\title{
A dynamic model for the Lagrangian stochastic dispersion coefficient
}

\author{
I. Pesmazoglou, ${ }^{\text {a) }}$ A. M. Kempf, ${ }^{\text {b) }}$ and S. Navarro-Martinez ${ }^{\text {c) }}$
}

(Dated: 1 December 2013)

A stochastic sub-grid model is often used to accurately represent particle dispersion in turbulent flows using Large Eddy Simulations. This type of models have a free parameter, the dispersion coefficient, which is not universal and is strongly grid-dependent. In the present paper, a dynamic model for the evaluation of the coefficient is proposed and validated in decaying homogeneous isotropic turbulence. The grid dependence of the static coefficient is investigated in a turbulent mixing layer and compared to the dynamic model. The dynamic model accurately predicts dispersion statistics and resolves the grid-dependence. Dispersion statistics of the dynamically calculated constant are more accurate than any static coefficient choice for a number of grid spacings. Furthermore, the dynamic model produces less numerical artefacts than a static model and exhibits smaller sensitivity in the results predicted for different particle relaxation times.

Keywords: LES, Mixing Layer, Stochastic Dispersion, Particles, Lagrangian, Dynamic Model

\section{INTRODUCTION}

A "particle" can be understood in its broadest sense as a solid, liquid or gaseous entity, with length scales much smaller than those of the "continuous" phase in which the particle is immersed; for example, droplets, solid particles, and bubbles. Particles understood in such manner can interact with each other and with the surroundings. Both types of interactions can become of central importance in numerous applications ranging from largescale chemical reactors and pulverized coal burners ${ }^{1}$, to the small-scale nanoparticle and soot formation and agglomeration in Internal Combustion Engines ${ }^{2}$ and Gas Turbines $^{3,4}$.

\section{A. A contextualization of the study: the need for sgs modelling in particle dispersion}

A number of experimental studies ${ }^{5-8}$ have investigated the characteristics of such two-phase flows, concluding that the dispersion process and resulting concentration field are highly dependent on particle size.

Reynolds averaged Navier-Stokes equation (RANS) modelling has been extensively used and is one of the dominant methods employed in industrial CFD codes. Numerical studies ${ }^{9-11}$ have shown that in turbulent multiphase flows the large scales dominate the dispersion process of the particulate phase. Although it is a useful tool in the area of particle dispersion, RANS creates complicated closure issues to model the effects of the larger more energetic scales. Arguably, recent work, as for example by Menter and Egorov ${ }^{12}$ on Scale Adaptive Simulations

\footnotetext{
a) Department of Mechanical Engineering, Imperial College, London SW7 $2 \mathrm{AZ}$

b) Chair of fluid dynamics CFD, Institute for Combustion and Gasdynamics, and Center for Computational Sciences and Simulation,Universität Duisburg-Essen

c) Department of Mechanical Engineering, Imperial College, London SW7 2AZ; Electronic mail: s.navarro@imperial.ac.uk
}

(SAS), has shown that RANS modeling may resolve energetic eddies using an improved length-scale equation, as derived by Rotta ${ }^{13}$, yet a Large Eddy Simulation (LES) treatment to obtain the energy spectrum of the unresolved sub grid-scales (SGS) is required.

On the other hand, Direct Numerical Simulation (DNS) involves the direct solution of the Navier-Stokes equations. DNS is an extremely powerful tool for fundamental studies ${ }^{14}$. However, it has a very high computational cost and it is currently infeasible to investigate large geometries and mixtures at high Reynolds numbers.

LES provides a good compromise between RANS and DNS, giving sufficient and relevant information for the motion of the dispersed phase. The main advantages of LES, apart from computational efficiency, are its ability to provide instantaneous values of the field and to directly resolve the most energetic eddies without a closure model. In the context of particle motion, Berrouk et al. ${ }^{15}$ have shown that LES demonstrates a superiority compared to RANS in predicting dispersion statistics.

A number of studies using LES have been carried out in the context of particle dispersion ${ }^{9,16,17}$, where it is assumed that the sub grid-scale effects are negligible for Stokes numbers much greater than one $(S t>>1)$. Miller and Bellan ${ }^{18}$ carried out an a priori analysis and concluded that for $S t \sim 1$ neglecting the sub grid-scale velocity fluctuations may lead to significant errors. Kuerten and Vreman ${ }^{19}$ used an a priori DNS and LES to simulate and compare turbophoresis in a particle laden flow. Fede and Simonin ${ }^{20}$ also used a DNS and a filtered-DNS in their study of unresolved fluid turbulence effects on the disperse phase and concluded that although the most energetic eddies are mainly responsible for the particle dispersion process, within a range of Stokes numbers $(0.5<S t<5)$ the sub grid turbulence acts as a random force which should be accurately taken into account. Shotorban and Mashayek ${ }^{21}$ concluded that including the unresolved velocities is critical when large filter sizes are employed and/or particles have a fast relaxation time. More recently, Berrouk et al. ${ }^{15}$, Bini and $\mathrm{Jones}^{22}$, Pozorksi and $\mathrm{Apte}^{23}$, and Pozorski et al. ${ }^{24}$ confirmed that a model to compensate for the sub grid continuous phase 
velocity fluctuations is required to reproduce the dispersion statistics of the particulate phase in an LES.

Summing up, one may say with sufficient certainty that the resolved LES velocity field cannot (on its own) accurately predict particle dispersion and particle velocity statistics. This is particularly relevant in situations with combustion, where the dispersion must be predicted correctly relative to other (gaseous, liquid, or solid) reactants. Therefore, a model to compensate for the effects of the unresolved scales is required. This study focuses on a Lagrangian stochastic model implementation for the incorporation of sub grid velocity effects on the particulate phase.

Alternative methodologies exist, such as the Approximate Deconvolution Method ${ }^{25}$ (ADM) proposed by Kuerten $^{26}$ and Shotoban and Mashayek ${ }^{27}$. ADM is a deterministic alternative to stochastic methods; the fluid velocity seen by the particle is de-convoluted in a series of steps using a filtering operator which approximates the inverse of the LES filtering function. Although it improves results for particle dispersion in a turbulent shear layer -compared to no use of a model- ADM cannot reconstruct scales smaller than the LES grid without the use of alternative deconvolution functions ${ }^{27}$.

\section{B. Incorporating the sub grid effects: The Lagrangian Stochastic Model}

A promising effort to incorporate the sub grid-scale contribution to the particle motion has been attempted with the use of Lagrangian Stochastic (LS) models. The LS models were initially developed for non-inertial fluid particle tracers in RANS but the implementation was readily extended to inertial particles. The methodology stems from a basic assumption for the evolution of the Lagrangian fluid velocity, namely that it is equivalent to a continuous Markov Process ${ }^{28}$.

In high Reynolds number flows the Lagrangian acceleration correlation is small over time increments much larger than the Kolmogorov time-scale and the Markovian assumption is justified. In that case, the changes of the velocity between two successive increments are nearly independent and depend only on local conditions.

The LS models have been extended to model the evolution of the dispersed phase concentration probability density function (pdf) in a turbulent flow both in RANS and LES studies. The LS models offer a great advantage to an Eulerian solution of the evolution of the particulate phase in that non-linear advection terms are implicitly included without approximation when following the particle motion ${ }^{29}$. Haworth ${ }^{30}$ and Pope ${ }^{31}$ offer an extensive review of pdf methods in turbulent flows including particle dispersion in a Lagrangian framework ${ }^{32}$.

Some fundamental work on LS models was conducted by Thomson ${ }^{33}$, who introduced suitable criteria for the selection of stochastic models to represent 'marked fluid particle' (i.e. non-inertial particle) trajectories in turbu- lent flows. Thomson argued that at a high Reynolds number, molecular diffusion effects can be considered negligible, and fluid particles can be assumed to travel with the local velocity of the fluid. Any changes in the concentration field occur due to the redistribution of fluid particles ${ }^{29}$. Therefore a correct description of the particle trajectories would allow the reconstruction of the concentration statistics, as for example the ensemble mean concentration field. He introduced the well mixed condition as the sole criterion required for the physical consistency of the model.

Using the Markov assumption for the evolution of the particle tracer velocity, a generalized Langevin stochastic differential equation (SDE) can be formulated ${ }^{28}$ :

$$
d \mathbf{v}_{f}=\mathbf{A}_{f} d t+\mathbf{B}_{f} \cdot d \mathbf{W}
$$

where $\mathbf{v}_{f}$ is the fluid particle Lagrangian velocity, $\mathbf{A}_{f}$ is the deterministic contribution to the acceleration, $\mathbf{B}_{f}$ is a diffusion tensor and $d \mathbf{W}$ is the incremental Wiener vector $^{28}$. The second term of equation (1) is the stochastic contribution to the fluid particle acceleration.

From the well mixed condition a suitable equation is derived for the deterministic component to the fluid particle acceleration $\mathbf{A}_{f}$ (also called the damping function, or drift term). The Markovian evolution of the particle velocity serves as the modeling assumption for the stochastic diffusion tensor $\mathbf{B}_{f}$.

For locally isotropic turbulence and time increments $d t$ larger than the Kolmogorov time-scale and within the inertial range of turbulence, Kolmogorov's similarity theory ${ }^{34}$ suggests that the statistics of $d \mathbf{v}_{f}$ take a universal form:

$$
<d v_{f}^{i} d v_{f}^{j}>=\delta_{i j} C_{o}^{*} \epsilon d t
$$

The Lagrangian velocity structure function of a fluid particle, equation (2), depends only on the time increment and the mean rate of dissipation of turbulent kinetic energy $\epsilon$, where $\delta_{i j}$ is the Kronecker delta. The constant of proportionality is the universal Kolmogorov constant $C_{o}^{*}$. The structure function is equivalent to the variance of the velocity and therefore its square root can be used as the standard deviation of the diffusion process of equation (1) i.e.

$$
d v_{f}^{i}=A_{f}^{i} d t+\sqrt{C_{o}^{*}} \epsilon d W^{i}
$$

At this point it is important to note two aspects of the LS model briefly described so far. Firstly, the model describes non-inertial particles in a RANS framework. The model compensates for the temporal fluctuations of the continuous phase velocity using a stochastic Lagrangian forcing quantified by the diffusion coefficient in equation (3). In the context of LES the stochastic contribution is required to simulate the unresolved turbulent scales, i.e. 
to compensate for the fluid phase spatial velocity fluctuations. Therefore, Kolmogorov's theory cannot be readily applied to derive the diffusion tensor $\mathbf{B}_{f}$ from first principles. On the other hand, models are heuristic and their constant of proportionality -equivalent to $C_{o}^{*}$ - is not universal, but is rather a modelling parameter scattered in the interval of $2-8$ depending on the test case ${ }^{32,35}$. The true particle trajectory is assumed to be approximated by a model trajectory.

The LS models described stem from the concept of a fluid particle, i.e. a notional parcel of fluid with a constant concentration. Physical particles are somewhat different as they may influence, or be influenced by the flow, due to their inertia or mass exchange with the continuous phase. Hereafter and unless stated otherwise, the term particle will be used to denote physical particles dispersed in a carrier fluid phase.

In a fair amount of literature the LS model for a fluid tracer is employed to acquire the fluid velocity at the particle location, i.e. the velocity 'seen' by the inertial particle $\mathbf{v}_{f} @^{21,23,24}$, or the incremental change of the unresolved fluid velocity seen by the particle ${ }^{20}$. One of the main problems arising from such LS-LES models is the evaluation of the eddy interaction time-scale seen by the particle and the dispersion coefficient $C_{o}^{*}$ required to evaluate the turbulent diffusion coefficient of equation (3). The aforementioned parameters exhibit a dependence on the Stokes number and the grid spacing and an effort has been made to fit empirical curves for the eddy interaction time-scale such as in the work of Jin et al. ${ }^{36}$. However, to the authors' knowledge, there has not been a comprehensive evaluation of the dispersion coefficient $C_{o}^{*}$.

The LS models mentioned thus far are somewhat different to the one employed in this study, the main difference being the fact that in the method of Bini and Jones $^{22,37,38}$, the stochastic contribution is directly accounted for in a SDE for the particle acceleration following a heuristic procedure. On the other hand, the aforementioned LS models use the Langevin equation to simulate the Lagrangian fluid phase velocity viewed by the particle starting from the Lagrangian description of the Navier-Stokes equations. Bini ${ }^{37}$ proposes an equation for the particle equation of motion which stems from the Lagrangian solution (Itô equivalent) of the spray-pdf equation of Williams ${ }^{39}$ (outlined in the following section). Bini's model has shown good agreement in particle acceleration statistics ${ }^{38}$ and dispersion characteristics in a turbulent shear layer ${ }^{22}$.

The present study aims at extending the applicability of this model by proposing a methodology for the dynamic evaluation of the dispersion constant $C_{o}$. The paper is structured as follows: First the methodology is introduced, followed by validation against a DNS database of particle laden shear-flow. Finally, the proposed model is compared with experimental data in a droplet-laden mixing layer; where the the results are compared with several static dispersion coefficients at different grid spac- ings.

\section{METHODOLOGY}

\section{A. Spray-pdf equation}

Williams ${ }^{39}$ introduced a conservation equation for the particle distribution function. The particle distribution function consists of the joint probability of the realization of $M$ variables that represent the state of the particulate phase (e.g. position, velocity, temperature, density, radius and their equivalent rates of change). In theory, all the microscopic variables relevant to the state of a particle within a two-phase flow should be taken into account. For example, in a flow with $N$ particles one would require an $N^{t h}$ order distribution function with $3 N$ velocity and spatial coordinates and a number of coordinates that would provide the particle size, shape, and their relevant rates of change. Such an exact solution would be intractable; an approximate model is therefore pursued by choosing a state vector $\boldsymbol{\Phi}$ consisting of a number of selected variables considered sufficient to represent the disperse phase in a given application (e.g. spray atomization). Any effects caused by the excluded microscopic variables can then be modelled by the addition of stochastic terms to the deterministic component of the Lagrangian equation for the rate of change of the property in question ${ }^{40}$.

For a set of $M$ independent variables the state vector of the $j^{t h}$ particle for a given location in space $\mathbf{x}$ and time $t$ is $\mathbf{\Phi}^{j}=\left\{\phi_{1}^{j}(\mathbf{x}, t), \ldots, \phi_{M}^{j}(\mathbf{x}, t)\right\}$. It is possible to characterize the random variables' phase space $\boldsymbol{\Psi}$ (with realizations $\boldsymbol{\Phi}^{j}$ for every $j$-indexed particle) of an ensemble of $N$ particles using the fine-grained density $\mathbb{F}(\boldsymbol{\Psi} ; \mathbf{x}, t)$, defined ${ }^{41}$ as:

$$
\mathbb{F}(\mathbf{\Psi} ; \mathbf{x}, t)=\sum_{j=1}^{N} \prod_{i=1}^{M} \delta\left[\psi_{i}-\phi_{i}^{j}(\mathbf{x}, t)\right]
$$

where $\delta$ is the Dirac delta function, $\psi_{i}$ the $i^{t h}$ random variable of the phase space, and $\phi_{i}^{j}$ is its realization by the $j^{\text {th }}$ particle. To clarify, a realization of the phase space means that a particle acquires a specific set of values for its state vector.

Now one may formulate an expression for the finegrained probability density function $\mathbb{P}(\boldsymbol{\Psi} ; \mathbf{x}, t)$ as the expectation of the fine-grained density function. Assuming that the particles are independently distributed and using the central limit theorem the expectation can be written as a sum over an infinite number of realizations (i.e. particles):

$$
\mathbb{P}(\mathbf{\Psi} ; \mathbf{x}, t)=\lim _{N \rightarrow \infty} \sum_{j=1}^{N} \prod_{i=1}^{M} \delta\left[\psi_{i}-\phi_{i}^{j}(\mathbf{x}, t)\right]
$$


To comply with the characteristics of a probability density, the fine grained pdf must be integrated to unity: a result that is approached as the number of realizations increases. The assumption of independently distributed particles has been used in the derivation of $\mathrm{Bini}^{37}$ for simplicity; Subramaniam ${ }^{42}$ has shown that it is not a necessary condition to define the pdf of the population of the particles. Finally, by differentiating (5) with respect to time following the motion of the phase space the evolution of the spray pdf can be obtained:

$$
\frac{\partial}{\partial t} \mathbb{P}(\boldsymbol{\Psi} ; \mathbf{x}, t)=-\sum_{i=1}^{M} \frac{\partial}{\partial \psi_{i}}\left[E\left[\left.\dot{\psi}_{i}\right|_{\mathbf{\Psi}=\boldsymbol{\Phi}}\right] \mathbb{P}(\mathbf{\Psi} ; \mathbf{x}, t)\right]
$$

where $E\left[\left.\dot{\psi}_{i}\right|_{\mathbf{\Psi}=\mathbf{\Phi}}\right]$ is the expectation of the rate of change of the random variable $\psi_{i}$ of the phase space $\boldsymbol{\Psi}$ conditioned upon the local state vector $\boldsymbol{\Phi}$. As mentioned, from the central limit theorem the expectation can be expressed as an ensemble average over independent realizations of the particle state vector.

In the context of LES, equation (6) is filtered spatially with a filtering kernel $G$ of width $\Delta$ as shown in equation (7). Where the filtering kernel has to be positive definite in order to maintain non-negative probabilities. In this way the pdf can be evaluated at the LES grid points and depends only on the local conditional filtered variables. Note that in the literature this equation is sometimes termed the filtered density function or filtered spray-pdf, for consistency, the term $p d f$ will be used here.

$$
\tilde{\mathbb{P}}(\mathbf{\Psi} ; \mathbf{x}, t)=\int \mathbb{P}\left(\boldsymbol{\Psi} ; \mathbf{x}^{\prime}, t\right) \cdot G_{\Delta}\left(\mathbf{x}, \mathbf{x}^{\prime}\right) d \mathbf{x}^{\prime}
$$

For a state vector with a single variable, here the particle velocity as a function of time and position $\mathbf{v}_{p}(\mathbf{x}, t)$, the following LES spray-pdf equation may be derived from the appropriate conservation equations for the evolution of the equivalent phase space random variable $\mathbf{V}_{p}(\mathbf{x}, t)^{37}$ :

$$
\frac{\partial \tilde{\mathbb{P}}\left(\mathbf{V}_{p} ; \mathbf{x}, t\right)}{\partial t}+\frac{\partial}{\partial \mathbf{V}_{p}}\left(a \tilde{\mathbb{P}}\left(\mathbf{V}_{p} ; \mathbf{x}, t\right)\right)=0
$$

The first term of equation (8) represents the local rate of change of the sub grid probability density in physical space. The second term incorporates the advection process due to the conditional rate of change ' $a$ ' of particle velocity. In general, the rate of change of the $j^{\text {th }}$ variable of the phase space $\dot{\psi}_{j}$ is conditioned upon the phase space being equal to the state vector (i.e. its realization) at any point within the filter volume $E\left[\left.\dot{\psi}_{j}\right|_{\Psi=\boldsymbol{\Phi}}\right]$. The conditional filtered particle acceleration is not readily available and depends on the full spectrum of turbulent motion; a model is therefore required for its closure.

\section{B. Lagrangian Stochastic Dispersion}

Itô's equivalent SDE for inertial particles is solved instead of the spray-pdf equation (8) following the solution proposed by Jones et al. ${ }^{22,38,43}$. Individual particle paths and velocities are computed from which the relevant fields can be calculated by ensemble averaging. Inertial effects are combined with the drag force, and it is assumed that the gravitational and buoyancy effects for Froude number of the particle are insignificant for $\mathrm{Fr}>>1$, and that thermo- and electro-phoresis can be neglected. With these assumptions, the particles' equation of motion can be described by the Lagrangian equations (9) and $(10)^{44}$.

$$
\begin{aligned}
d \mathbf{x}_{p} & =\mathbf{v}_{p} d t \\
\frac{d \mathbf{v}_{p}}{d t} & =\frac{\mathbf{U}_{f}-\mathbf{v}_{p}}{\tau_{I}}
\end{aligned}
$$

where $\mathbf{x}_{p}, \mathbf{v}_{p}$ are the position and velocity vectors of the particle, $\mathbf{U}_{f}$ is the gas velocity, and $\tau_{I}$ is a time-scale representative of the inertial effects governed by the assumed type of forcing between the gaseous and disperse phases.

The RHS of equation (10) is equivalent to a single realization of the acceleration $a$ (i.e. the acceleration of one particle) in the spray-pdf equation (8). One may notice that it is a function of the full spectrum of the gaseous velocity, which can be decomposed into a filtered and sub-filter component $\left(\mathbf{U}_{f}=\tilde{\mathbf{U}}_{f}+\mathbf{U}_{s g s}\right)$. By equivalence to a stochastic diffusion process ${ }^{28}$, the influence of the unresolved SGS velocity fluctuations, $\mathbf{U}_{s g s}$, on the particle acceleration is modeled by adding a stochastic forcing to the resolved/deterministic component of (10).

Miller and Bellan ${ }^{18}$ and Okong'o and Bellan ${ }^{45}$ proposed that for a scalar, this contribution can be related to the realization of a normally distributed random variable with a standard deviation proportional to the sub grid scalar fluctuation.

Jones and $\mathrm{Bini}^{22,38}$ model the unresolved contribution by a Wiener term taken as $d W=\xi \sqrt{d t}$, where $\xi \sim \mathcal{N}(0,1)$, and scaled by a 'turbulent' diffusion coefficient; see equation (11). By dimensional analysis this coefficient is expected to be proportional to the time- and length-scales of the unresolved stresses quantified by the sub grid kinetic energy, $k_{s g s}$, and the time-scale of turbulent interactions between the two phases, $\tau_{t}$. This results in:

$$
d \mathbf{v}_{p}=\frac{\left(\tilde{\mathbf{U}}_{f}-\mathbf{v}_{\mathbf{p}}\right)}{\tau_{p}} d t+\sqrt{C_{o} \frac{k_{s g s}}{\tau_{t}}} d \mathbf{W}
$$

where, $C_{o}$ is the dispersion constant. Following $\mathrm{Bini}^{38}$, the unresolved kinetic energy of the gas phase is calculated as: $k_{s g s}=2 \Delta^{2} C_{s}^{4 / 3} \tilde{S}_{i j} \tilde{S}_{i j}$ where $\tilde{S}_{i j}$ is the filtered strain tensor, $\tau_{p}$ finally is the particle relaxation time: 


$$
\tau_{p}^{-1}=\frac{3}{4} \frac{\rho_{f} C_{D}}{\rho_{p} d_{p}}\left|\tilde{\mathbf{U}}_{f}-\mathbf{v}_{\mathbf{p}}\right|
$$

where $\rho_{p}$ and $\rho_{f}$ are the particle and continuous phase densities, $d_{p}$ is the particle diameter, and $C_{D}$ is the drag coefficient. The relaxation time $\tau_{p}$ indicates the time it will take for a particle to adjust to a change in the carrier phase velocity $\tilde{\mathbf{U}}_{f}$ (or to come to rest in a stationary flow).

The drag coefficient, $C_{D}$, is evaluated using an empirical relation to the particle Reynolds number $R e_{p}=$ $\left(\rho_{p} d_{p}\left|\tilde{\mathbf{U}}_{f}-\mathbf{v}_{\mathbf{p}}\right|\right) / \mu$ (where $\mu$ is the viscosity of the continuous phase), defined by the drag law of Yuen-Chen ${ }^{46}$ assuming spherical particles.

$$
C_{D}= \begin{cases}\frac{24}{R e_{p}}\left(1+\frac{R e_{p}^{2 / 3}}{6}\right) & 0<R e_{p}<100 \\ 0.424 & R e_{p}>1000\end{cases}
$$

For the solution of the above equations the continuous phase properties at a given particle's position are provided from the Eulerian LES simulation using a bi-linear interpolation. The turbulent time scale, $\tau_{t}$, proposed by Bini and Jones ${ }^{38}$ depends on the constant $\alpha$, which determines the degree of non linearity introduced in the model.

$$
\tau_{t}=\tau_{p}\left(\frac{\tau_{p} k_{s g s}^{1 / 2}}{\Delta}\right)^{2 \alpha-1}
$$

Following a detailed evaluation of the parameter $\alpha$, Bini and Jones ${ }^{22}$ concluded that a value of 0.8 is required to produce suitable levels of kurtosis in turbulent flow acceleration pdfs. The limits of $\alpha=0$ and $\alpha=1$ correspond to normal and log-normal pdfs of acceleration, respectively ${ }^{38}$. Finally, a proportionality constant is required for the stochastic diffusion term, in this case $C_{o}$, which scales the stochastic contribution to the velocity of the Lagrangian particles.

The ensemble average of all particle paths should give the Eulerian equivalent of the positions and velocities of the particles, $\mathbf{U}_{p}=\left\langle\mathbf{v}_{p}>_{n}\right.$, where the brackets $<>$ indicate averaging over an ensemble of $n$ particle paths. Hereafter, unless stated otherwise, upper case letters will be used to represent Eulerian velocities and lower case for the Lagrangian velocities.

\section{Dynamic model for the evaluation of the dispersion coefficient}

The expression (11) is heuristic and uses the Markovian assumption to model the sub grid contribution with a Brownian diffusion term. The scaling of the Lagrangian forcing to the sub-grid kinetic energy is controlled by $C_{o}$.
However, this dispersion coefficient cannot be related to the Kolmogorov constant arising from the velocity structure function ${ }^{33}$. Moreover, $C_{o}$ cannot be evaluated analytically from a spectral analysis as performed by Lilly ${ }^{47}$ for the Smagorinsky constant.

Results of Jones et al. ${ }^{43}$ have shown that the choice of $C_{o}$ influences the dispersion process and in this work it is shown that the coefficient exhibits significant grid and particle relaxation time dependence.

As in the turbulent stress model implementation of Piomelli ${ }^{48}$, a test-filtering process is suggested by convolution of a LES generic variable $\tilde{\phi}$ with a test filtering kernel $G_{\hat{\Delta}}$, viz:

$$
\hat{\tilde{\phi}}(\mathbf{x})=\int \tilde{\phi}\left(\mathbf{x}^{\prime}\right) \cdot G_{\hat{\Delta}}\left(\mathbf{x}, \mathbf{x}^{\prime}\right) d \mathbf{x}^{\prime}
$$

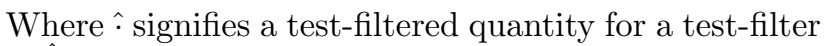
size $\hat{\Delta}=2 \Delta$. At this point, one must make a clarification in terms of what is test-filtered in the context of the proposed model. The drift and diffusion terms of the SDE, equation (11), are functions of local interpolated LES Eulerian quantities $\left(\tilde{\mathbf{U}}_{f}, k_{s g s}\right)$ and Lagrangian particle properties. The test filter is applied to all the Eulerian quantities used for the calculation of the drift and diffusion terms prior to their interpolation to the local particle position.

For simplicity $\hat{\imath}$ will imply both test-filtered and testfiltered derived quantities, i.e. $\widehat{d \mathbf{v}_{p}}$ is the change in Lagrangian particle velocity due to its interaction with the test-filtered Eulerian field within an increment $\widehat{d t}$ (where $\hat{\imath}$ is included to specify that the integration is over the time-step dictated by the CFL condition applied to the test-filtered Eulerian velocity field).

Similarly the test-filtered drift $\hat{\mathbf{A}}$ and diffusion $\hat{B}$ terms are functions of the interpolated test-filtered Eulerian values $\left(\widehat{\hat{\mathbf{U}}_{f}}, \widehat{k_{s g s}}\right)$, the test-filter derived relaxation and turbulent interaction time-scales $\left(\widehat{\tau}_{p}, \widehat{\tau}_{t}\right)$, and the Lagrangian filter-independent quantities i.e. the particle velocity $\mathbf{v}_{p}$ and mass $m_{p}$. Therefore, one may formulate an equation for the test-filtered derived acceleration:

$$
\begin{aligned}
\widehat{d \mathbf{v}}_{p} & =\frac{\left(\widehat{\tilde{\mathbf{U}}}_{f}-\mathbf{v}_{\mathbf{p}}\right)}{\widehat{\tau}_{p}} \widehat{d t}+\sqrt{C_{o} \frac{\widehat{k_{s g s}}}{\widehat{\tau}_{t}}} d \mathbf{W} \\
& =\hat{\mathbf{A}} \widehat{d t}+\sqrt{C_{o}} \hat{B} d \mathbf{W}
\end{aligned}
$$

It must be stressed that, unless stated otherwise, the parameters in (16) are not explicitly test-filtered, but are derived from the Eulerian test-filtered quantities. Also note that for simplicity, in the derivation that follows the dispersion coefficient has been taken out of the diffusion term $\hat{B}$. The diffusion and drift terms could then be replaced without any loss of generality to a phenomenological, SDE based, model different to the one proposed by $\mathrm{Bini}^{37}$. 
Having established the filtering process, one must formulate an assumption on which the comparison (between LES filtered and test-filtered quantities) is based. The evident difficulty in direct comparison of acceleration arises from the Wiener process. Since it is stochastic in nature with a zero mean, the ensemble average acceleration of all particles within a given cell should not be a function of the diffusion coefficient (given the number of samples/particles is sufficiently high).

In the present work it is assumed that the ensembleaveraged change in kinetic energy of particles, $\left\langle d K_{p}\right\rangle$, with same initial conditions within a cell should be the same for the two filtering levels (Eulerian LES and the a posteriori test-filtered equivalent). Physically, this means that the total energy transfer from the Eulerian to the Lagrangian phase within a given time increment should be grid independent and only a function of the flow characteristics.

Equation (17) gives the kinetic energy of a single particle, which at a time $t_{0}$ is the same for the two filtering levels:

$$
K_{p}=\frac{1}{2} m_{p} \mathbf{v}_{p}^{2}=\frac{1}{2} m_{p}\left(v_{p_{1}}^{2}+v_{p_{2}}^{2}+v_{p_{3}}^{2}\right) .
$$

To calculate the variation of particle kinetic energy $d K_{p}$ from $t_{0}$ to $t_{0}+d t$ it should be noted that the kinetic energy is a function of the random variable $\mathbf{v}_{p}$, which is continuous but not differentiable in time. Therefore traditional calculus cannot be employed, and the varia- tion of kinetic energy is retrieved using Itô's Lemma for a time-dependent function of a stochastic process:

$$
\begin{aligned}
d K_{p}=\left[A_{i} \cdot \frac{d K_{p}}{d v_{p_{i}}}\right. & \left.+\frac{1}{2} C_{o} B^{2} \frac{d^{2} K_{p}}{d v_{p_{i}}^{2}}\right] d t \\
& +\sqrt{C o} B \frac{d K_{p}}{d v_{p_{i}}} \cdot d W_{i}
\end{aligned}
$$

Taking the ensemble average of equation (18) and assuming a large number of particles, such that $<$ $d \mathbf{W}>_{n} \rightarrow 0$ the following equations can be written for the variation of particle phase kinetic energy derived from the LES (19) and test-filtered (20) velocities:

$$
\begin{aligned}
\left\langle d K_{p}\right\rangle & =\left\langle\left[m_{p} A_{i} \cdot v_{p_{i}}+\frac{3}{2} m_{p} C_{o} B^{2}\right] d t\right\rangle \\
\left\langle\widehat{d K_{p}}\right\rangle & =\left\langle\left[m_{p} \widehat{A}_{i} \cdot v_{p_{i}}+\frac{3}{2} m_{p} C_{o} \widehat{B}^{2}\right] \widehat{d t}\right\rangle
\end{aligned}
$$

Note that all the terms in the RHS of (18) are evaluated at $t_{0}$; therefore the Lagrangian particle velocity and mass is the same for the two filtering levels. Moreover note that because the diffusion tensor is isotropic, the summation over all directions reduces to the second terms of the RHS of equations (19) and (20). Equating (19) and (20) for a common time increment one may formulate the following expression for the dynamically derived dispersion coefficient $C_{d y n}$ :

$$
C_{d y n}=\left|\frac{\left\langle m_{p}\left[v_{p_{1}}\left(f_{r} A_{1}-\hat{A_{1}}\right)+v_{p_{2}}\left(f_{r} A_{2}-\hat{A_{2}}\right)+v_{p_{3}}\left(f_{r} A_{3}-\hat{A_{3}}\right)\right]\right\rangle}{\left\langle\frac{3}{2} m_{p}\left(\hat{B}^{2}-f_{r} B^{2}\right)\right\rangle}\right|
$$

Where the factor $f_{r}$ relates the time increments of the two differential equations to compare the transfer of kinetic energy from the Eulerian phase to the particulate phase in the same time-step. For simplicity this timestep is chosen, to be that from the LES, for which values are readily available. The CFL number ${ }^{49}$ is assumed to be the same for both filters, and therefore the factor $f_{r}=\widehat{d t} / d t=\hat{\Delta} / \Delta=2$. The absolute value in equation (21) is taken to enforce a positive constant, since its square root is required for the evaluation of the SDE equation (11). Similar to the Germano model for the evaluation of the Smagorinsky constant, the dispersion coefficient is test-filtered to ensure smoothness.

Although the methodology for the evaluation of $C_{d y n}$ is similar to that of the dynamic Germano model, $C_{d y n}$ does not have to acquire a value of 0 in a laminar flow (unless $\tilde{U}_{f} \approx v_{p}$ ); instead $C_{d y n}$ has to attain a finite value. It can be shown that in the limit of the test filtered relative velocity approaching the filtered relative velocity
$\hat{\tilde{U}}_{f} \rightarrow \tilde{U}_{f}, C_{d y n}$ is proportional to $\alpha\left(\tilde{U}_{f}-v_{p}\right)^{2(1-\alpha)}$. Yet, the stochastic contribution at the filtering level in the limit of a laminar flow $\sqrt{C_{d y n} k_{s g s} / \tau_{t}} \rightarrow 0$ will vanish as $k_{s g s} \rightarrow 0$. These results were confirmed on a laminar mixing layer (not shown).

\section{VALIDATION OF THE DYNAMIC MODEL}

\section{A. Configuration and Modelling Parameters}

For the purpose of validating the model the simple DNS test case of Ahmed and Elghobashi ${ }^{50}$ was chosen. It consists of a uniform shear flow, where decaying homogeneous isotropic turbulence is superimposed to the constant shear. The carrier phase is subsequently seeded with particles to measure their dispersion statistics.

Figure 1 depicts the configuration of the test case. The domain is periodic in $x$ and $z$, and a shear-periodic 


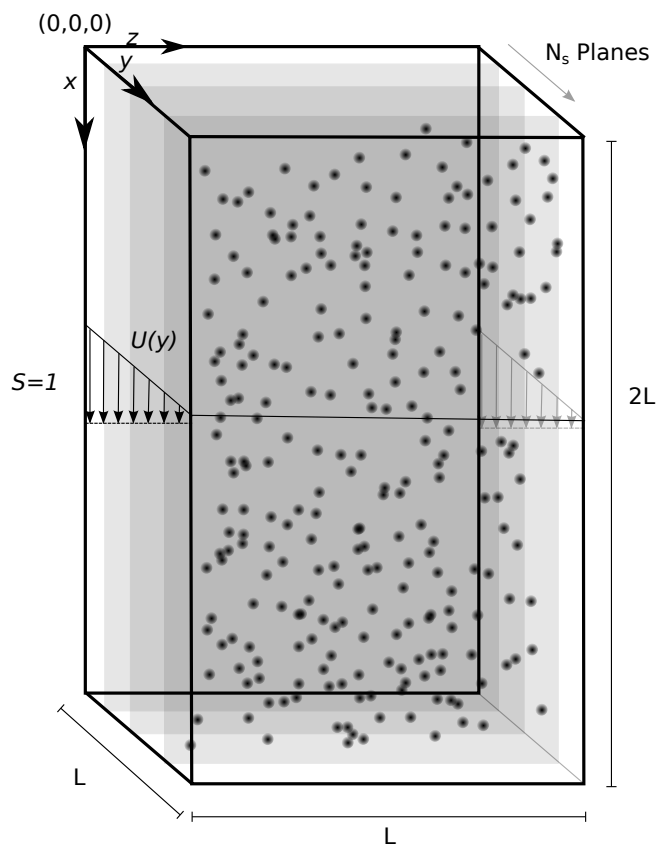

FIG. 1. Schematic diagram of the case of Ahmed and Elghobashi $^{50}$. A constant shear, $S$, is applied in the $y$-direction to which a decaying homogeneous isotropic turbulent field is superimposed. $22^{2}$ particles are seeded on each of the $N_{S}=22$ planes placed at equidistant positions in the direction of the shear.

boundary condition is used in the direction of the imposed mean velocity gradient $(y)$. Details for the implementation of the shear-periodic condition can be found in Gerz et al. ${ }^{51}$.

The dimensionless velocity gradient $S$ is equal to 1 for a unity length scale $L$ and velocity difference $\Delta \bar{U}_{f}$, where the over-bars signify temporal means. The velocity in $x$-direction at a given location is derived by the mean shear and superimposed turbulence, viz:

$$
U_{f, 1}(x, y, z)=S y+U_{f, 1}^{\prime}(x, y, z)
$$

where $U_{f}^{\prime}$ is the turbulent velocity field. The initial turbulent velocity fields, $U_{f}^{\prime}$ are generated from the prescribed initial energy spectrum, $E_{0}(k)$,

$$
E_{0}(k)=\frac{16}{2 \pi^{1 / 2}} \frac{3}{2} U_{0}^{r m s} \frac{k^{4}}{k_{p}^{5}} \exp \left(-2\left(k / k_{p}\right)^{2}\right)
$$

where $k_{p}=5$ is the wave number around which the maximum energy is concentrated. The initial root mean square velocity is $U_{0}^{r m s}=0.03530$, which gives an initial total kinetic energy of $E_{T 0}=0.0018696$. The initial Integral and Kolmogorov length scales are $l_{0}=0.072980$ and $\eta_{k, 0}=0.006761$ respectively. The initial turbulent Reynolds number based on the integral length scale is $R e_{t}=24.5$. All quantities are dimensionless; normalized with the mean velocity difference $\Delta \bar{U}_{f}$ and length scale $L$.

The simulation solves the continuous phase in an Eulerian LES using the in-house PsiPhi code ${ }^{52,53}$. The initial turbulent fields where generated from the prescribed energy spectrum (23) using the HIT3D pseudo-spectral $\operatorname{code}^{54}$. The velocity fields were a-posteriori filtered into an LES with $80 \times 40 \times 40$ cells for the domain dimensions $2 L \times L \times L$ (see Fig 1). This is equivalent to a ratio of $\Delta / \eta_{k, 0}=1.85$ suggesting that more than $90 \%$ of the energy spectrum is resolved.

The unknown sub grid-scale stresses of the momentum equation are closed using the Smagorinsky eddy-viscosity model $^{55}$ with the model constant of $C_{s}=0.173$ suggested by Lilly ${ }^{56}$. A Central Differencing Scheme second order accurate in space is used for the convective momentum fluxes; and a third order Runge-Kutta Scheme is used for integration in time. The time-step width is set according to the CFL condition ${ }^{49}$, with $\mathrm{CFL}=0.3$. The code uses implicit filtering with equally sized cubic cells, thus ensuring numerical accuracy and efficient parallelization.

The peak skewness, $S k_{u}$, based on the gaseous velocity derivatives is defined as:

$$
S k_{u}(t)=\sum_{i=1}^{3} \frac{-\frac{1}{3}\left\langle\left(\partial u_{i} / \partial x_{i}\right)^{3}\right\rangle}{\left[\frac{1}{3}\left\langle\left(\partial u_{i} / \partial x_{i}\right)^{2}\right\rangle\right]^{3 / 2}}
$$

where $\langle\cdot\rangle$ implies ensemble averaging over all the cells of the domain. The first peak in $S k_{u}$ (at approximately $t=$ 1 ) is used as the particle injection criterion, following the same conditions as the DNS studies ${ }^{50}$. The peak signifies that a fully developed turbulence is established ${ }^{50}$.

Therefore, at this time particles are initialized on $N_{s}=$ 22 planes positioned at equidistant locations in the $y$ direction. On each $(x, z)$ plane, $N_{p s}=22^{2}$ particles are seeded randomly with initial velocities equal to those of the uniform phase. A total of $N_{p}=22^{3}$ particles are tracked using equation (10) for the DNS of ${ }^{50}$ from which equation (11) is derived and used in the LES of this work. Two different particle sizes are considered. The first case consists of particles with $d_{p}=40 \mu \mathrm{m}$ (case A) and the second with $d_{p}=125 \mu \mathrm{m}$ (case B). The ratio of disperse to continuous phase density is the same for both cases and equal to $\rho_{p} / \rho_{f}=19000$. Ahmed and Elghobashi ${ }^{50}$ assume a constant particle relaxation time, un-correlated to the particle relative velocity, of $\tau_{p}=0.1$ and $\tau_{p}=1.0$ for the first and second case respectively. For consistency, their definition for the particle relaxation time is also used here.

The particle positions are tracked in time and their 
mean-square displacement, $\left\langle x_{p_{i}}^{2}(t)\right\rangle$, is calculated using:

$$
\begin{array}{r}
\left\langle x_{p_{i}}^{2}(t)\right\rangle=\frac{1}{N_{p}} \sum_{j=1}^{N s} \sum_{k=1}^{N_{p s}}\left\{\left[x_{p_{i}}(t)-x_{p_{i}}\left(t_{0}\right)\right]_{k}\right. \\
\left.-\left[\left\langle x_{p_{i}}(t)\right\rangle\right]_{j}\right\}^{2}
\end{array}
$$

where $\langle\cdot\rangle$ imply ensemble averaging over all particles, and with

$$
\left[\left\langle x_{p_{i}}(t)\right\rangle\right]_{j}=\frac{1}{N_{p s}} \sum_{k=1}^{N_{p s}}\left[x_{p_{i}}(t)-x_{p_{i}}\left(t_{0}\right)\right]_{k} .
$$

Particles released in different planes have different initial velocities due to the imposed mean shear. To remove this bias from the dispersion variance, the corresponding mean displacement $\left[\left\langle x_{p_{i}}(t)\right\rangle\right]_{j}$ of particles released from a given plane $j$ is subtracted as shown in (25). The displacement variance takes into account only the effects of turbulence ${ }^{50}$.

\section{B. Results}

Figure 2 presents the variation of the total kinetic en$\operatorname{ergy} E_{T}(t)$ (bottom), and skewness $S k_{u}(t)$ (top), of the Eulerian phase as a function of time. Good agreement was found with the DNS values of the total kinetic energy. The initial dip in $E_{T}$ can be attributed to a faster dissipation rate compared to the forcing on large scales induced by the mean shear. As the flow evolves the Integral and Kolmogorov length scales increase in size and the cascade of energy from the forced wave numbers proceeds ${ }^{50}$. The rate of dissipation of the - now increased $-\eta_{k}(t)$ is lower than the energy input from the mean shear effecting in the gradual increase of the $E_{T}$. The slight underestimation of the LES- $E_{T}$ can be attributed to the cut-off of the filtering process resulting to a -nearly constant- loss of the order of $5 \%$ which is in accordance with the assumption that the grid spacing chosen resolves over $90 \%$ of the energy spectrum.

The Skewness of the velocity derivatives, $S k_{u}$, follows a similar trend to the DNS results with peaks and throughs dampened. The dampening can also be attributed to the filtering process as velocity gradients are smoothened over the domain due to the reduced resolution compared to this of the DNS. A first peak is observed at approximately $1 \mathrm{~s}$ after the simulation is initialized. At this time instance the particles are injected in the domain with the Eulerian phase velocities.

Figure 3 presents the displacement variance for the two particle sizes in cases A (small particles) and B(large particles). Statistics were available for directions $x$ and $z$, and four particle transport methodologies where used for each case, namely: no dispersion model $C_{o}=0$, static coefficient with values $C_{o}=1,2$, and the dynamic model for $C_{d y n}$.

For both cases, the span-wise displacement variance, $\left\langle z_{p}^{2}\right\rangle$, is an order of magnitude lower than this of the axial

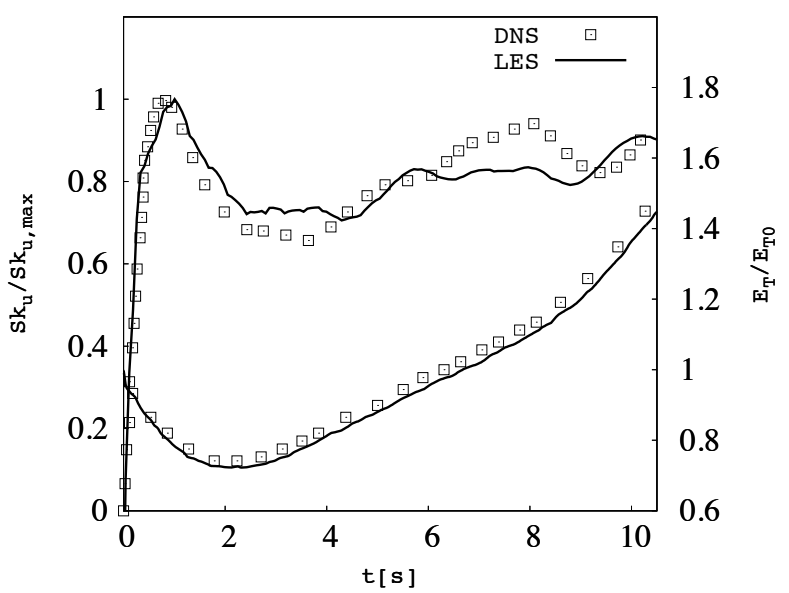

FIG. 2. Temporal variation of the normalized Skewness (top) and Total Kinetic Energy (bottom), for our LES and the DNS of Ahmed and Elgobashi ${ }^{50}$.

direction. Results for case A nearly collapse on the same line for all dispersion implementations. Similarly for case $\mathrm{B}$ there are small visible differences in the various models, the common trend being to produce good results for case A and slightly over-estimate the dispersion levels in case B.

The effect of modelling the sub grid-scale contribution becomes visible for the dispersion variance in the axial direction. Ignoring the SGS contribution to the particle transport $\left(C_{o}=0\right)$ results to a significant underestimation of the mean dispersion variance. The value of a constant dispersion coefficient has no analytical solution, as discussed in the previous section, but it is suggested ${ }^{37,57}$ that it lies in the range of $0.5-2$. For this particular grid spacing, it seems that the $C_{o}=1$ would be the most appropriate choice. The dispersion levels are overestimated for both the light (case A) and heavier (case B) particles with $C_{o}=2$.

As a first order validation, the proposed dynamic model performs very well. The dispersion levels produced are closest to the DNS equivalent for both cases. When the dynamic model is employed, the solution $\left\langle x_{p}^{2}(t)\right\rangle$ lies between the two static choices of $C_{o}=1$ and 2. Such boundedness in conjunction with the produced levels of dispersion suggests that the phenomenological assumption of the model is sound.

The two cases of figure 3 suggest that dispersion results depend on particle diameter and consequently relaxation time. For light particles, the $C_{o}=1$ and $C_{d y n}$ results are very similar, however for heavier particles there is a noticeable divergence. In the Lagrangian Stochastic model of Bini and Jones ${ }^{22}$ the diffusion term is a function of the particle relaxation time, which depends on the particle diameter, and the assumption of linear scaling of $C_{o}$ for all diameters is made intrinsically. In the present work, the dynamic coefficient is calculated from 

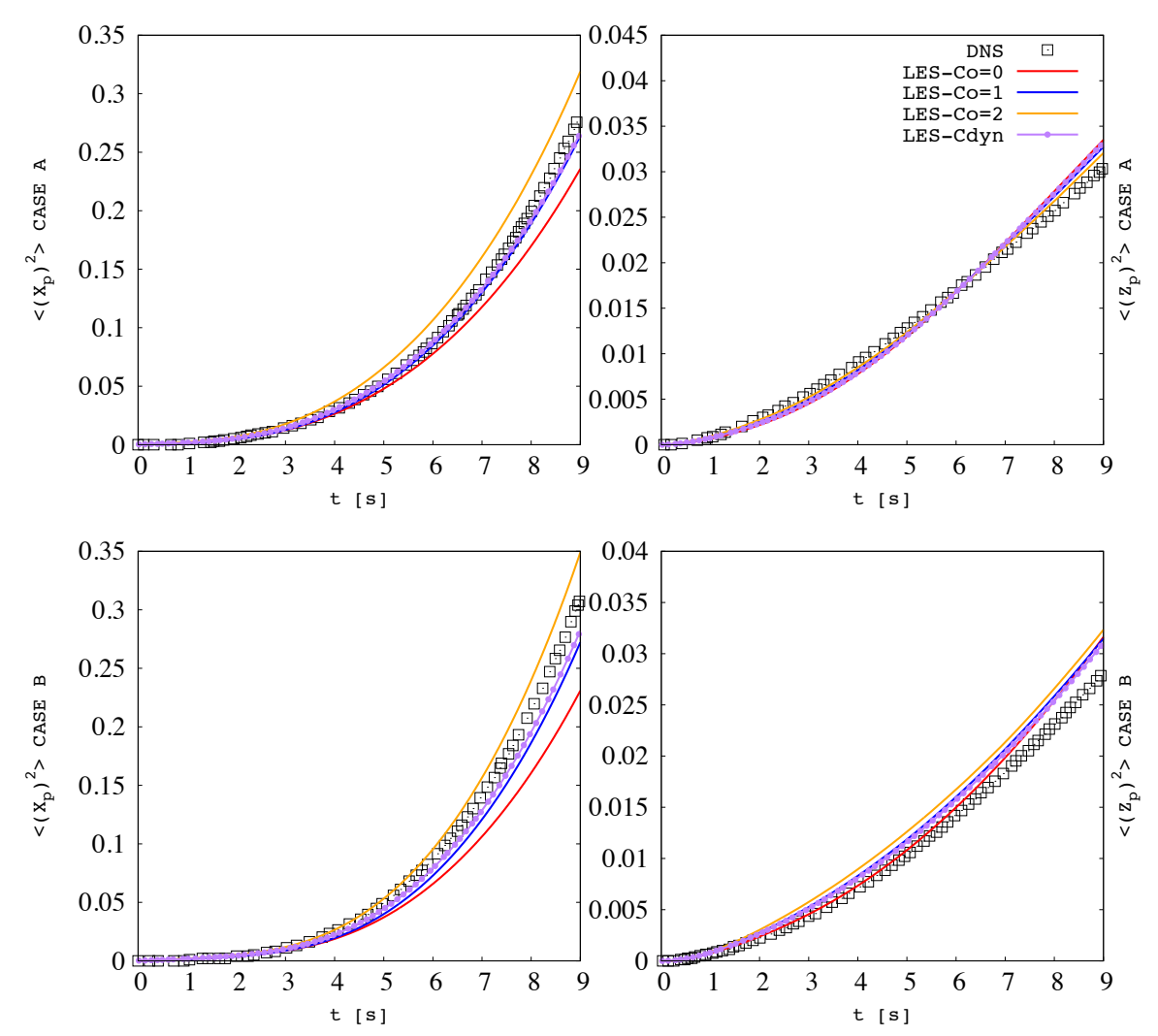

FIG. 3. Temporal variation of the displacement variance in directions $x$ and $z$ compared to the DNS of ${ }^{50}$ (points) for case A (top) and case B (bottom).

a local mass weighted ratio, (21), making its evaluation size-dependant.

From the results shown, it can be concluded that the correct prediction of particle motion requires a model to incorporate the sub-grid effects. The dynamic model accurately predicts the dispersion variance of the disperse phase. Moreover, the size-weighting of the dynamic coefficient results in smaller variations in the dispersion statistics between particles of different size compared to the static model.

\section{INVESTIGATING GRID DEPENDENCE OF $C_{o}$ IN A SHEAR MIXING LAYER:}

\section{A. Experimental Configuration and Modelling Parameters}

In this section, the static and dynamic model grid dependence in steady state statistics (temporally averaged dispersion levels) are investigated. A mixing layer seeded with particles in chosen for the investigation. The mixing layer thickness grows monotonically in the axial direction and therefore the 'resolution' of the mixing layer increases linearly (provided a constant grid spacing is used). This configuration enables the investigation of the dispersion model's performance in scenarios of not fully-resolved shear with regards to temporally-averaged dispersion levels.

The case considered here was studied experimentally by Tageldin and Cetegen ${ }^{58}$, and numerically by Jones et $a l .57$. It consists of a shear mixing layer produced by two parallel air flows within a $200 \times 150 \times 150 \mathrm{~mm}$ domain in the $x, y$, and $z$ directions respectively as depicted in figure 4 . The two flows have a bulk velocity of $U_{\text {slow }}=2$ and $U_{\text {fast }}=7.1 \mathrm{~ms}^{-1}$. The two streams are separated prior to the test section with a splitter plate. Honeycomb straighteners and mesh screens are used to create a uniform velocity profile for each stream. Wires of $1.5 \mathrm{~mm}$ diameter are used to trip the boundary layers produced on either side of the splitter plate in order to achieve self-similarity in a shorter distance. A Reynolds number, based on air properties and momentum thickness of $R e_{\theta}=225$ was measured at the splitter plate.

The fast stream is homogeneously seeded with water droplets with diameters ranging from 2 to $50 \mu \mathrm{m}$ with a number mean diameter of approximately $15 \mu \mathrm{m}$ as shown in figure 5. The Stokes number for the mean particle diameter varies from $S t=0.08$ near the exit of the domain to $S t=1.2$ in the near field of the mixing layer ${ }^{57,58}$. The resulting spray can be considered dilute, with a volumetric void fraction of the order of $5.5 \cdot 10^{-6}$; therefore, droplet interactions are considered negligible. In addi- 


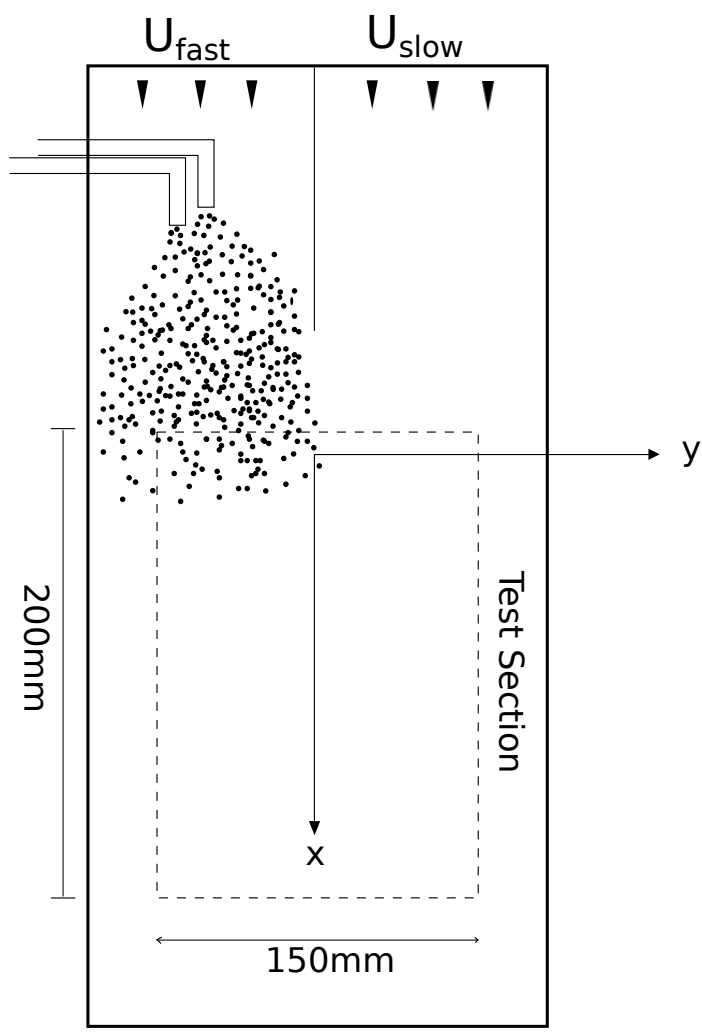

FIG. 4. Experimental configuration of ${ }^{58}$. Particles are seeded in the fast stream.

tion, the streams are at ambient temperature and evaporation rates are assumed to be very small. An average concentration of 4 million droplets in the domain is conserved by injecting the number of droplets that have left the domain upstream of the mixing layer.

Fully developed turbulence is considered with an intensity of $2 \%$ and $4-5 \%$, in the fast and slow streams respectively, based on the mean velocity of the droplet seeded stream. The Kolmogorov length- and time-scales were estimated from the experiment ${ }^{58}$ to be $\eta_{k}=0.15 \mathrm{~mm}$ and $\tau_{k}=0.2 \mathrm{~ms}$ respectively in the shear layer.

The same LES PsiPhi code is used with the parameters mentioned in Section III. Pseudo-turbulent inflow boundary conditions are created with Klein's inflow generator ${ }^{59,60}$ with the experimental values of $\mathrm{rms}$ velocities and length-scales, The computational time varied from 180 to $5000 \mathrm{CPU}$ hours for the finest case.

For the investigation of the effects of the dispersion constant $C_{o}$, two well-documented downstream spreading measures where considered: the mixing layer thickness, $\delta_{0.8}$, and the momentum layer thickness, $\theta_{u}$. The dimensionless velocity $u^{*}$ is defined:
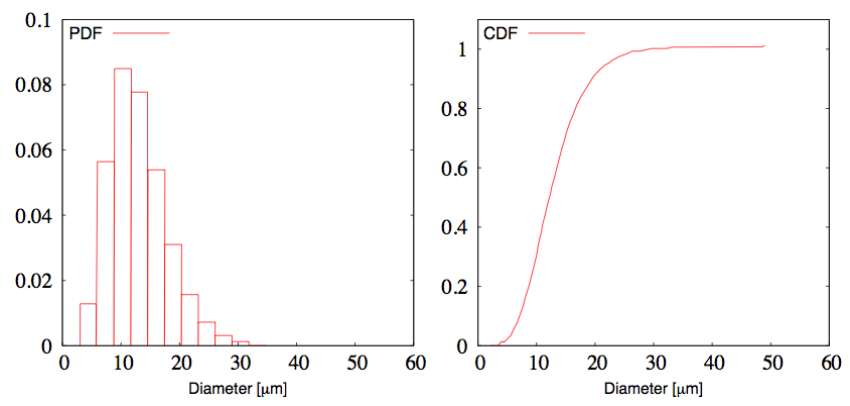

FIG. 5. Probability Density (PDF) and Cumulative Density (CDF) functions of the particle diameter in the test section of the flow.

$$
u^{*}=\frac{U_{p}-U_{\text {slow }}}{U_{\text {fast }}-U_{\text {slow }}}
$$

From which the thicknesses $\delta_{0.8}$ and $\theta_{u}$ are defined as:

$$
\begin{gathered}
\delta_{0.8}(x)=\left.y\right|_{u^{*}=0.1}-\left.y\right|_{u^{*}=0.9} \\
\theta_{u}(x)=\int_{-\infty}^{\infty} u^{*}\left(1-u^{*}\right) d y
\end{gathered}
$$

For the definition of $u^{*}$ the Eulerian particle velocity field, $U_{p}(x, y)$, constructed from the Lagrangian particle velocities $\mathbf{v}_{p}$ (see Section B), is used. For a given cell, the average velocity of all particles passing through it in a given time interval $\Delta T_{n}$ is calculated. The time $\Delta T_{n}$ is a generic temporal interval equal to $2000 \times \Delta t$, where $\Delta t$ is the simulation time-step width. The final Eulerian particle velocity $U_{p}$ is calculated by averaging the values from each temporal interval.

The mixing layer thickness $\delta_{0.8}$ at a given axial location $x$, is defined as the position on the $y$-axis where the particle normalized velocity equals to 0.1 , minus the position where it is 0.9 (for the co-ordinate system shown in figure 4). Therefore, the mixing layer thickness is a positional measure on a velocity basis.

The momentum layer thickness (29) definition of Tageldin and Cetegen ${ }^{58}$ is retained to allow for direct comparison with the experimental results. Evaluating $\theta_{u}$ involves the integration over the entire range of cells that contain particles, i.e. the entire particle domain. This integration method is used to avoid negative values of momentum thickness at positions where there are no particles (i.e. $U_{p}=0$ ) since the normalizing velocity, $U_{\text {slow }}$, used in the experiment is constant and positive.

For the normalized velocity plots of both the gaseous and fluid phases the dimensionless height $y^{*}=(y-$ $\left.y_{0.5}\right) / \theta_{u}$ is used. It must be noted here that for the normalization of $y^{*}$ the same baseline momentum thickness 

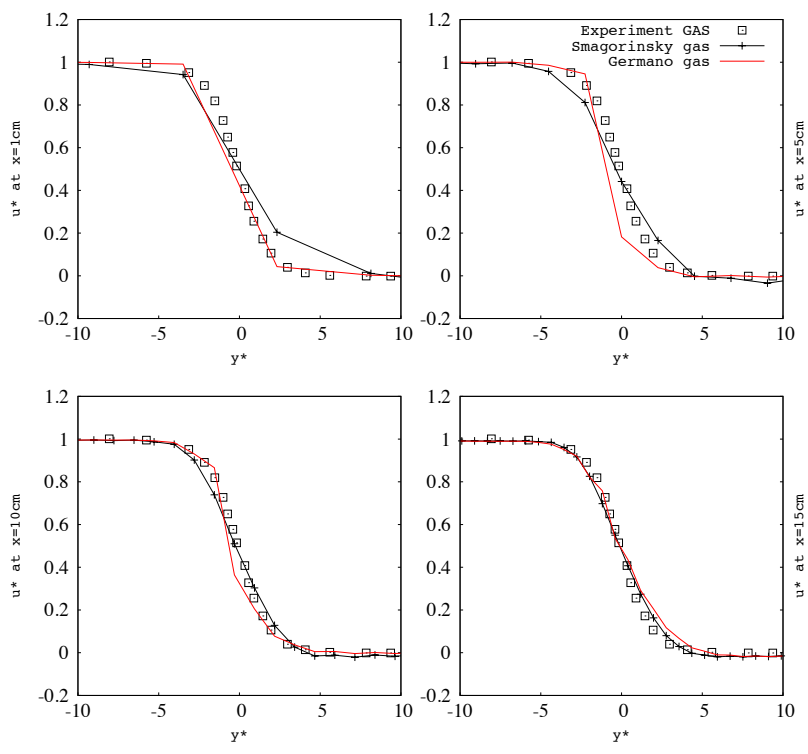

FIG. 6. Comparison of the Smagorinsky model and the Germano model for the evaluation of $C_{s}$ in a medium grid spacing $(\Delta=2 \mathrm{~mm})$ simulation.

is used for all the different $C_{o}$ cases at a given grid spacing. The reason for the choice of a constant normalization is to allow for a direct comparison of the different velocities of the various $C_{o}$ cases.

\section{B. Results}

The discussion of the results begins by analyzing the effects of the static dispersion coefficient $C_{o}$ on the momentum and mixing layer thickness for the fine $(0.8$ and $1.5 \mathrm{~mm})$ and coarse $(2 \mathrm{~mm})$ grid cases. To investigate the sufficiency of the Smagorinsky model, a simulation using the dynamic Germano implementation of Piomelli and $\mathrm{Liu}^{48}$ was carried out for a grid spacing with $\Delta=2 \mathrm{~mm}$, showing that the Smagorinsky model produces adequate results for the continuous phase (figure 6). In addition to a reduced computational cost, the Smagorinsky model allows a direct evaluation of the sub grid kinetic energy. Moreover, the influence of a dynamic $C_{s}$ on the particle dispersion coefficient is removed, facilitating the investigation of the grid spacing dependence of the stochastic dispersion coefficient $C_{o}$. Therefore, the Smagorinsky model was used for the remaining simulations.

Figure 7 shows instantaneous snapshots of a passive scalar to represent the mixing process in the test section for different grid spacings. The scalar is equivalent to a mixture fraction, and is transported using the LES code with boundary conditions of 1 for the slow stream and 0 for the fast stream. One may note the ability of the LES to capture instantaneous fields, but also

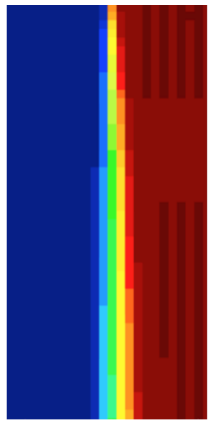

(a)

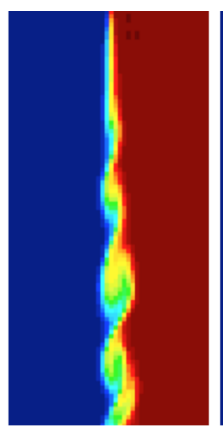

(b)



(c)

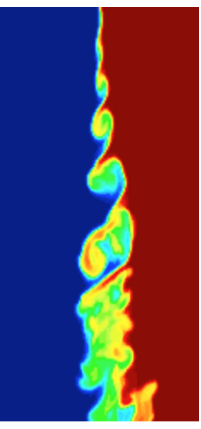

(d)
FIG. 7. Instantaneous snapshot of a passive scalar with: (a) $\Delta=4 \mathrm{~mm}$, (b) $\Delta=2 \mathrm{~mm}$, (c) $\Delta=1.5 \mathrm{~mm}$, (d) $\Delta=0.8 \mathrm{~mm}$. A passive scalar to illustrate the mixing process is assigned as 1 in the slow stream (red) and 0 in the fast stream (blue).

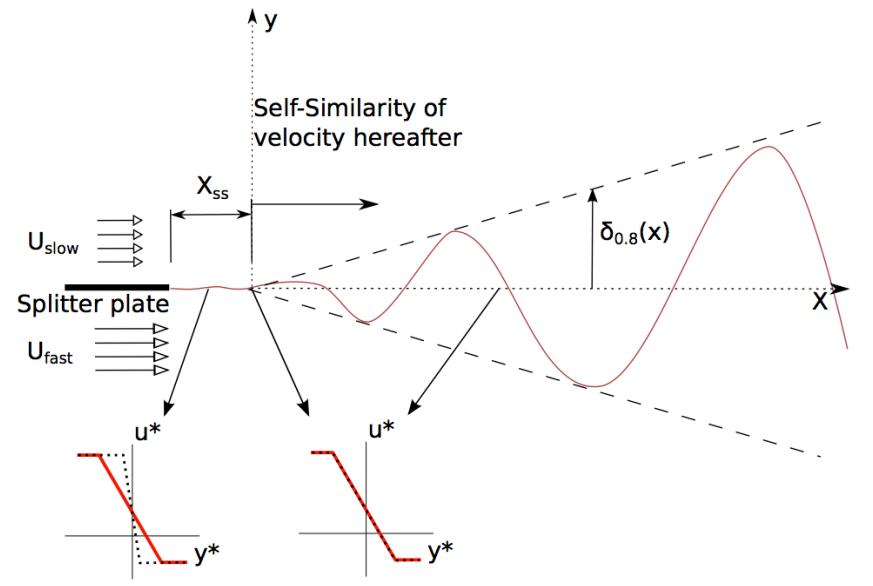

FIG. 8. The position where self-similarity is reached for the velocity (red line in normalized sketches) and a linear mixing layer thickness, $\delta_{0.8}(X)$, starts developing, is strongly dependent on the transition length, $X_{s s}$. A virtual origin, $X$, is set at the position, $X_{s s}$, where the dotted line of normalized velocites $u^{*}$ coincides with the self similar solution (red line).

the pivotal effect of the grid spacing on the flow. The $4 \mathrm{~mm}$ and $0.8 \mathrm{~mm}$ filter widths are arguably the equivalent of a poorly-resolved and a well-resolved LES respectively; in the coarsest $\Delta / \eta_{k} \approx 26.6$ and in the finest mesh $\Delta / \eta_{k} \approx 5.3$. Due to the low resolution of the $4 \mathrm{~mm}$ filter width results are presented for the remaining cases.

Present simulations with $\Delta / \eta_{k} \leq 7.25$ will resolve more than $80 \%$ of the energy spectrum in an equivalent homogenous isotropic turbulence and can therefore be considered good LES as suggested by Pope ${ }^{31}$. Although this result must be taken with caution as we are considering shear flow, it suggests that both fine meshes are well resolved and the coarsest mesh is not.

From figure 8 , it can be seen that the position where self-similarity in velocity is reached is defined as the transition length, $X_{s s}$. This depends on the grid spacing 
for numerical simulations, and the flow configuration and splitter plate properties in the experiment. The ability to predict the exact distance $X_{s s}$ requires a highly resolved simulation of the splitter plate and experimental configuration, which is outside the scope of this work. Therefore, experimental data are compared to the self-similar results. A normalized dimensional value $X=x-X_{s s}$ is employed, where $X_{s s}$ is used to compensate for the splitterplate-to-test-section distance and the position where self similarity is reached, and $x$ is the absolute distance from the inflow of the simulation.

\section{Dispersion with a static coefficient $C_{o}$}

Figure 9 shows plots of the momentum and mixing layer thicknesses increasing with normalized axial position. For the two fine cases (figures $9(\mathrm{a}-\mathrm{b})$ ), the mixing layer is better predicted when the stochastic dispersion is included (i.e. $C_{o}>0$ ) whereas the $C_{o}=0$ cases underpredict the two thicknesses (apart from the position furthest upstream). The stochastic contribution is higher on the $1.5 \mathrm{~mm}$ grid than on the $0.8 \mathrm{~mm}$ grid.

The momentum thickness exhibits a significant correlation with $C_{o}$ seen mainly in the pre-developed region of the flow (where self-similarity of the gas-phase velocity has not been reached, i.e. $x<X_{s s}$ ). Once the flow reaches a self-similar solution the effect of $C_{o}$ decays as exhibited by the converging lines for $C_{o}=0-8$ in figure $9 a_{2}$.

On the coarser grid ( $\Delta=2 \mathrm{~mm}$ ) it is found that the effect of $C_{o}$ has a decisive role on the dispersion. It can be seen that a stochastic dispersion contribution must be employed to give results near the experimental levels. Such results are expected since a wider range of scales are unresolved, which contribute to the sub grid-transport. Therefore, the lack of a model will cause an underestimation of the particle dispersion.

Addition of the model overestimates the mixing layer, but provides fairly accurate levels for the momentum thickness. Such a result can be understood by considering the definitions of the two thicknesses. The mixing layer thickness is defined on a positional basis by calculating the cell where the averaged particle velocity fulfills the conditions of (28), and therefore the error is directly proportional to the grid spacing. On the other hand, the momentum thickness is based on the velocity.

In the pre-developed region, the effect of $C_{o}$ is to increase/enhance momentum. Particles move from interface between the streams to the top (slow) stream with a higher vertical velocity than in lower or zero $C_{o}$ cases. The sharp velocity gradient of the flow upstream translates into high turbulent diffusion of the particles, increasing $C_{o}$ enhances this effect on particle motion, as the stochastic contribution increases with the static coefficient. The velocity gradient seen by a particle is steeper, inducing a higher stochastic contribution. Depending on the magnitude of the vertical particle velocity (governed by $C_{o}$ ) and the relaxation time $\tau_{p}$, the particles will readjust downstream to the velocity of the flow. Downstream, the overall stochastic contribution decreases (as the gas velocity gradient smoothens) and the dispersion levels of the various static $C_{o}$ cases converge.

Figures 10 and 11 illustrate the "numerical advancement" of the particle velocity distribution. The downstream over-prediction of $\theta_{u}$ apparent in Fig. 9 may be understood by comparing the normalized velocity plots for the two fine grids (Figs. 10-11). The LES gas velocity of the $\Delta=0.8 \mathrm{~mm}$ case converges to the self-similar solution of the experimental data faster than that of the $\Delta=1.5 \mathrm{~mm}$ grid. In addition, it is noticeable that the effects of the model constant differ depending on the position of the flow. Upstream, it is clear that for higher values of $C_{o}$, particles have moved further into the slow stream. In the $\Delta=0.8 \mathrm{~mm}$ cases the deeper penetration of the particles is only clear up to $x=50 \mathrm{~mm}$, whereas for the $1.5 \mathrm{~mm}$ cases it is clearly visible even at $x=150 \mathrm{~mm}$.

Finally, note the negative values of the normalized velocities in the results of the experiment and simulations, signifying that the particle velocity $U_{p}$ is less than $U_{\text {slow }}$ in the slow stream. Depending on the particle relaxation time and vertical velocity component, the particle axial velocity gradually adjusts to the free stream velocity downstream. The tails of the normalized velocity plots tend to $u^{*}=-U_{\text {slow }} /\left(U_{\text {fast }}-U_{\text {slow }}\right)$, i.e. where there are no particles and $U_{p}=0$.

Similar patterns are observed in the development of the two thicknesses in the coarser grid. In the cases of Fig. 9(c-d) the numerical overshoot is much higher. Arguably, this is an outcome of the inability of the coarse grid to capture flow phenomena. Fig. 12 reveals that the mixing layer is captured by a few cells only. Therefore the velocity gradients between each cell are much steeper, (with respect to cell spacing not physical space), causing high sub grid shear levels and thus increasing the momentum thickness, while at the same time also increasing the positional error of the mixing layer thickness.

From the normalized velocities of Fig. 12, the effect of the dispersion coefficient is clear in the tails of the profiles. The penetration level increases proportionally to $C_{o}$. Lack of a stochastic dispersion model influences the coarse cases in that particles do not move into the slow stream; the $C_{o}=0$ lines lead the other cases until they intersect them. This intersection is caused by the steeper particle velocity gradient, which translates into narrower mixing and momentum layer thicknesses.

From the analysis thus far we can argue that a model to incorporate the sub grid-scale effects must be employed to produce the observed dispersion levels. Both the mixing and momentum layer thicknesses require $C_{o}>1$ for all grid spacings. Secondly, it can be argued that the choice of $C_{o}$ is not universal. It seems that the optimal dispersion coefficient is subject to the grid spacing. 

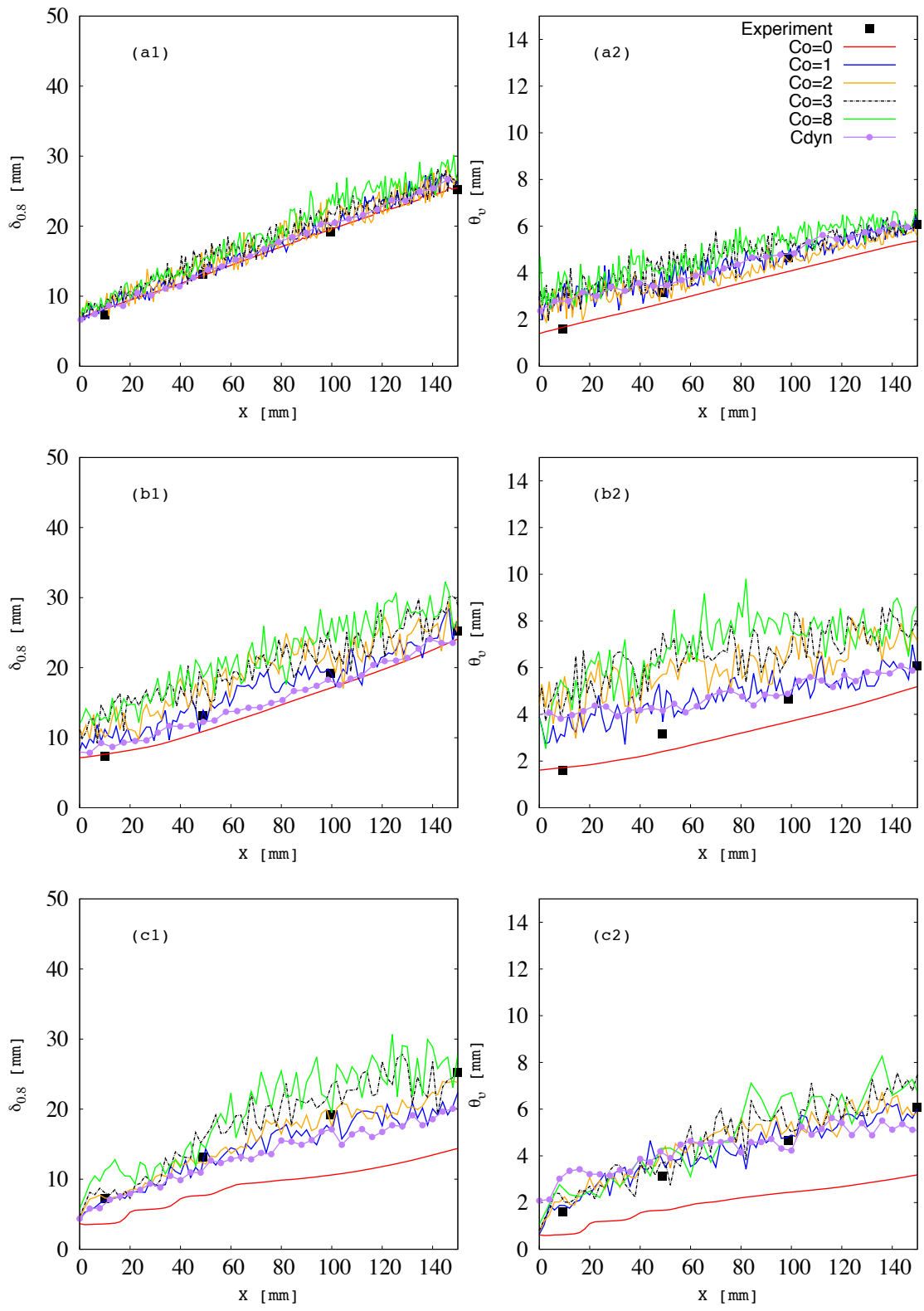

FIG. 9. Mixing $\left(\delta_{0.8}\right.$, left $)$ and momentum $\left(\theta_{u}\right.$, right $)$ thicknesses versus normalized axial position $X$ for different dispersion coefficients $C_{o}=0-3,8:$ (a) $\Delta=0.8 \mathrm{~mm}$, (b) $\Delta=1.5 \mathrm{~mm}$, (c) $\Delta=2 \mathrm{~mm}$

\section{Dispersion with a dynamic coefficient $C_{o}$}

In the finest case of $\Delta=0.8 \mathrm{~mm}$, shown in Fig. 9a, the dynamic model produces accurate dispersion levels for the mixing and momentum thicknesses. Arguably, the mixing layer thickness is similar to the simulation without a model for the sub grid-scale, but the $C_{o}=0$ case underestimates the momentum thickness. By comparing the two thicknesses shown in Figs. $9 a_{1}$ and $9 a_{2}$, one may conclude that even though the particles reach the correct dispersion levels, the velocity distribution is underestimated in the $C_{o}=0$ case. It is clear that the use of the dynamic model provides better results than with $C_{o}=0$.

Moreover, it is interesting to note that for the same post processing methodology the simulations using a dynamic model for the evaluation of the dispersion coefficient have a smaller fluctuation amplitude about the mean mixing and momentum layer thickness when compared to any choice of non-zero static dispersion coefficient, for any grid size. Such a result suggests a faster convergence to the correct dispersion levels compared to the static model implementations. One may therefore conclude that a dynamic dispersion field can enhance stability by varying the stochastic contribution depending on local flow characteristics, as opposed to a global static 

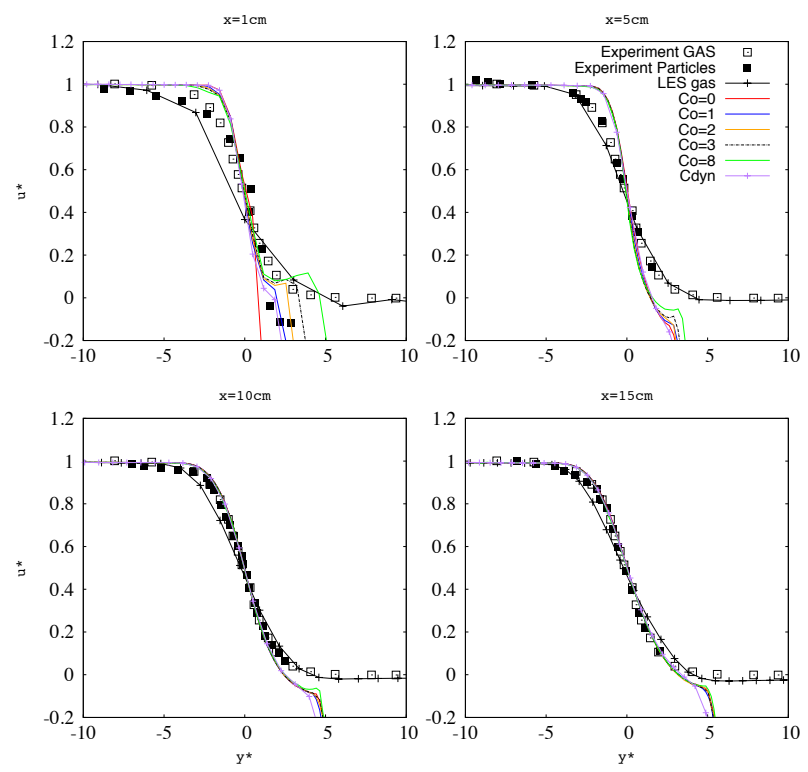

FIG. 10. Normalized velocity $u^{*}$ versus normalized height $y^{*}$ for $\Delta=0.8 \mathrm{~mm}$ for different dispersion coefficients and LES gas filtered velocity. Measurements are shown for $x=$ $1,5,10$ and $15 \mathrm{~cm}$ in the axial direction and compared with the experimental results of ${ }^{58}$. The same baseline momentum thickness at a given position is used for all the different $C_{o}$ cases and the dynamic coefficient $C_{d y n}$.
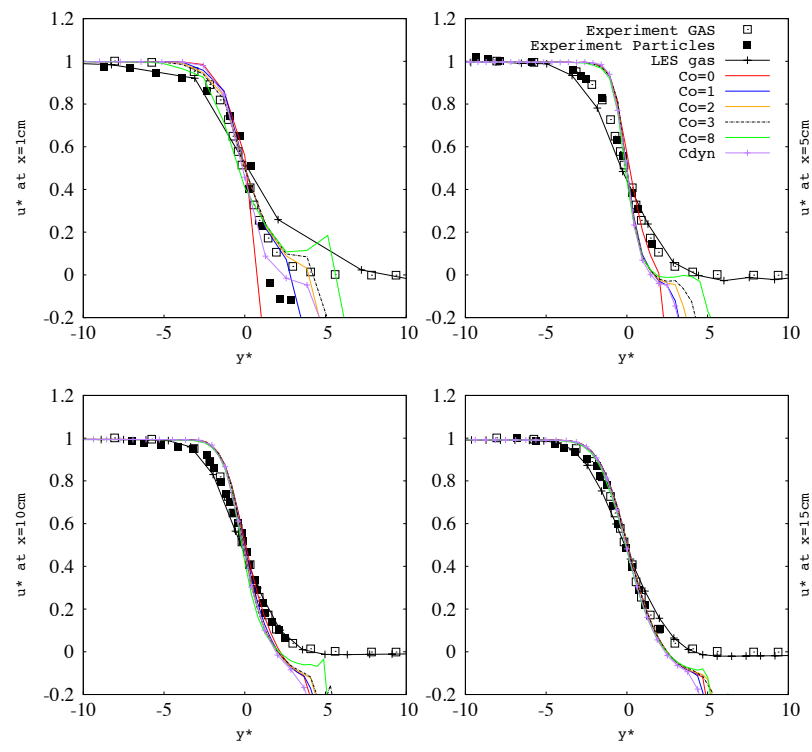

FIG. 11. Normalized velocity $u^{*}$ versus normalized height $y^{*}$ for $\Delta=1.5 \mathrm{~mm}$ for different dispersion coefficients and LES gas filtered velocity.
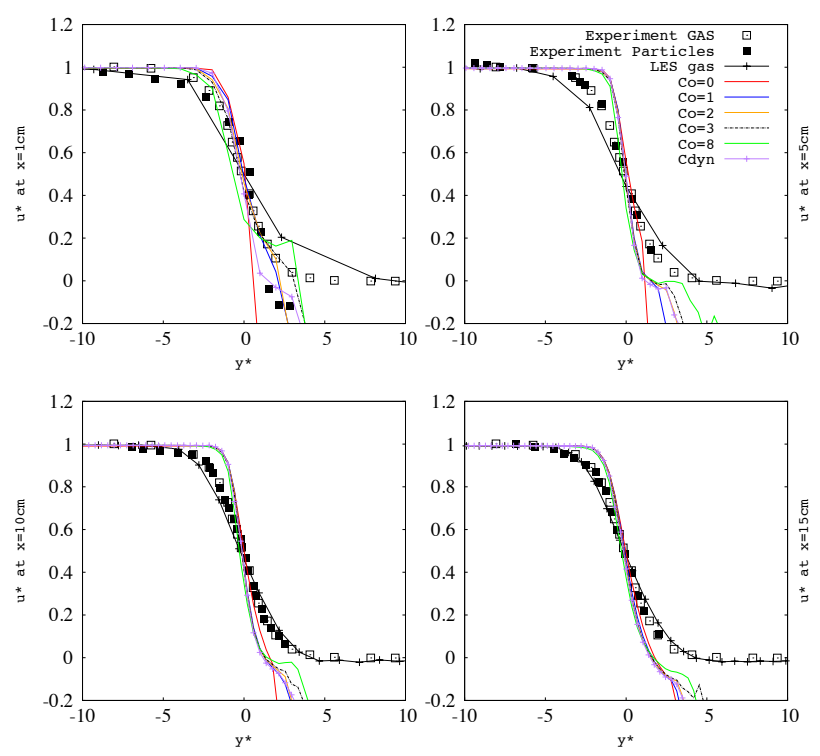

FIG. 12. Normalized velocity $u^{*}$ versus normalized height $y^{*}$ for $\Delta=2 \mathrm{~mm}$ for different dispersion coefficients and LES gas filtered velocity.

$C_{o}$ choice. Most importantly, it seems that the dynamic model can predict the dispersion coefficient at various locations in the flow. Some over-predictions are still observed at upstream positions, but this may be attributed to the fact that the gas phase shear is only resolved by a few cells.

For $\Delta=1.5 \mathrm{~mm}$, depicted in Fig. 9b, the dynamic model produces comparatively better results than any choice of static coefficient. The difference between no model and the dynamic model in terms of the two thickness measures is greater compared to that of the finer mesh. The momentum layer thickness, with the exception of the first point, is considerably underestimated in the simulation without a stochastic dispersion model. Equivalently the mixing layer thickness is also underestimated when the sgs are not incorporated.

At a grid spacing of $2 \mathrm{~mm}$ the dynamic model gives good agreement, though, closer agreement was found overall in the static model with $C_{o}=2$. However, from Fig. 9c the dynamic model still gives significantly better results than the simulation with $C_{o}=0$, which highly under-estimates dispersion levels for both mixing and momentum layer thicknesses. Moreover, the dynamic model produces less 'noisy' results suggesting faster convergence relative to any $C_{o}>0$ case. Thirdly, it can be argued that dynamic models tend to fail as the grid spacing becomes too coarse, as both the filter and test-filter widths need to be within the turbulence inertial range to allow for a valid comparison between, the two rates of change of particle kinetic energy.

Figures 13 and 14 show the average absolute and 

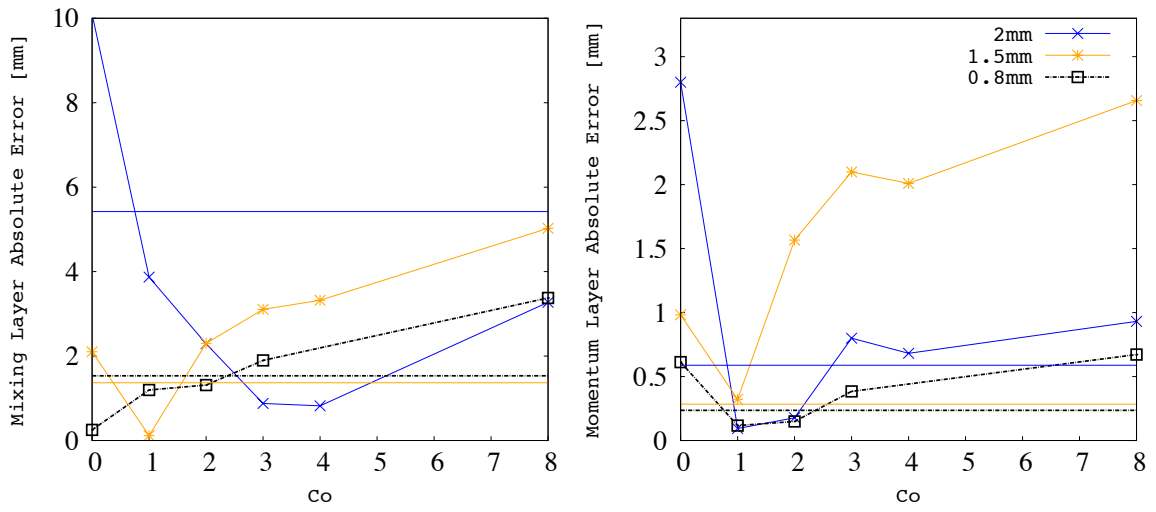

FIG. 13. Mixing and momentum layer thickness absolute errors versus the dispersion coefficient Co, for various grid spacings $\Delta$. The solid line for each grid spacing represents the absolute error of the dynamic model. The error is a mean difference between the simulated and experimental results in the range of $x=80-150 \mathrm{~mm}$. It can be observed that the use of a dispersion model decreases the levels of error for all grid spacings. The dynamic dispersion coefficient produces good results compared to a static $C_{o}$.
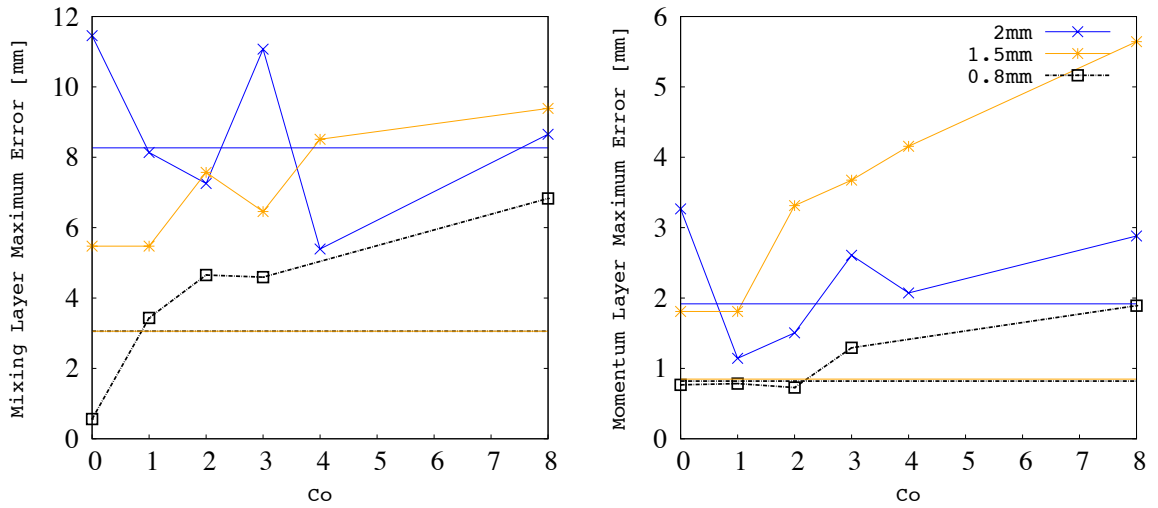

FIG. 14. Mixing and momentum layer thickness maximum errors versus the dispersion coefficient Co, for various grid spacings $\Delta$. The solid line for each grid spacing represents the maximum error of the dynamic model. The error represents the maximum deviation of the simulations from the experimental results in the range of $x=80-150 \mathrm{~mm}$. It can be observed that the use of a dispersion model $\left(C_{o}>0\right)$ decreases the maximum error for coarse $(\Delta=2 \mathrm{~mm})$ and increases it for finer $(\Delta=0.8,1.5 \mathrm{~mm})$ grid spacings. The dynamic dispersion coefficient produces good results compared to a static $C_{o}$.

maximum errors in the mixing and momentum layer thicknesses. The average error was calculated as the mean deviation between simulation results and linearlyinterpolated experimental data. The on/off effect of the dispersion coefficient is obvious, as in all cases the mean error decreased when the model was implemented. After this initial drop there are slight fluctuations which do not suggest a monotonic behavior, i.e. a constant decrease of error as $C_{o}$ increases, yet it may be suggested that with regards to the momentum layer a minimum error is achieved for $C_{o} \approx 1$.

The dynamic model predicts better results for both mixing and momentum thickness compared to simulations without a stochastic dispersion model. Absolute errors are lower than the the majority of choices of a static coefficient and are close to the minimum error produced by the investigatory choice of $C_{o}$.

An additional advantage of the dynamic model arises when investigating the maximum error of the two thicknesses. This error measurement represents the maximum deviation from the expected experimental dispersion statistics. Figure 14 shows that the inclusion of a dispersion model affects the maximum error differently depending on the grid spacing.

The dynamic model has lower mixing layer thickness maximum errors compared to all non-zero static dispersion coefficient choices for the finest cases and coarsest case. Secondly, the level of maximum deviation from the experimental data is similar for the 0.8 and $1.5 \mathrm{~mm}$ grid spacings, for both the momentum and mixing layer 
thicknesses. For $\Delta=2 \mathrm{~mm}$, the dynamic model produces lower maximum errors than the $C_{o}=0$ simulation, but it does not give the best results. Arguably, however, the error levels are similar to those produced by the static coefficients on the same grid spacing.

We believe that the main reason for which the dynamic results are less 'noisy', and provide lower levels of maximum error lies in its flow adaptive calculation. As opposed to the static coefficient, the dynamic method enhances or reduces the dispersion coefficient according to the local flow conditions. Consequently, using the proposed methodology one may obtain a more stable solution for the particle dispersion statistics.

From what follows, the dynamic coefficient can be represented by an Eulerian field. The instantaneous ensemble averages of $C_{d y n}$ are calculated for each Eulerian node at every time-step. Therefore, a time averaged $C_{d y n}$ can be readily calculated.

Figure 15 depicts the instantaneous (particle ensembleaveraged) and time-averaged dynamic coefficient distribution for the different grid spacings investigated. Figure 15 a depicts the average and instantaneous $C_{d y n}$ field of the finest grid spacing. The Eulerian fields presented clearly show that the coefficient is far from constant. The instantaneous plots have a structure that clearly indicates a correlation with the flow vortices. The level of resolution is directly related to the values of the $C_{d y n}$ field.

Figure 16 shows the variation of the dynamic dispersion coefficient in the $y$-direction at $x=100 \mathrm{~mm}$. The dispersion coefficient acquires its highest value in the fast stream where the turbulent rms velocity is higher comparatively to the the slow stream. An increase in the grid spacing from 1.5 to $2 \mathrm{~mm}$ results in a $30 \%$ increase in $C_{d y n}$ in the fast stream, as opposed to the (more than) threefold increase from the 0.8 to $1.5 \mathrm{~mm}$ grid spacing.

Within the mixing layer, unlike a turbulent stress dynamic model, there is a sharp drop in the dispersion coefficient. It is the whole stochastic diffusion term that increases and not the coefficient in particular. What the model suggests is that the increase in $\sqrt{k_{s g s} / \tau_{t}}$, due to the sharp velocity gradient, is in fact over-predicted, thus requiring a smaller dispersion coefficient to realistically represent the energy transfer from the gaseous phase to the particulate phase. This has an additional dissipative effect, which can be related to the reduced fluctuations of the results from the dynamic model. Given the same flow conditions, a constant $C_{o}$ would over-predict the local energy transfer, thus 'shooting' the particle with a higher velocity towards the slow stream. The particle would then reach the next cell and, depending on the local conditions, its velocity will re-adjust.

\section{CONCLUSIONS}

The Lagrangian Stochastic model of Bini and Jones $^{22,37}$ has previously shown very good predictions for particle velocity and acceleration statistics. However, the dispersion constant was found to be very grid dependent.

In this work, using similar assumptions to scale similarity models, we have derived a model that dynamically evaluates the dispersion coefficient. The method uses a test-filtering process for the Eulerian variables of the continuous phase to derive a test-filtered equivalent particle acceleration SDE. Assuming that the average rate of change of the particle kinetic energy does not vary with the filter width for a given time increment, an equation for the dispersion coefficient was derived.

The model was first validated on a shear flow with decaying homogenous turbulence, where the uniform phase was seeded with particles. LES predictions of particle dispersion statistics were compared with DNS data. It was shown that using a model to incorporate SGS effects is required for the correct prediction of particle motion. The dynamic model accurately predicts the dispersion variance of the particle phase of two different particle sizes.

For the investigation of grid dependence and temporally-averaged dispersion statistics, a series of LES of a droplet-laden mixing layer were carried out with different grids and static dispersion coefficients. The mixing layer and momentum layer thicknesses are underpredicted without a model, and the results suggested that the stochastic dispersion model is required for an accurate prediction of the experimental results. The static coefficient model increases the particle accelerations depending on the grid spacing, and consequently the levels of motion intended to represent the unresolved scales. In both fine and coarse grids the normalised velocities of the particulate phase at upstream locations approach the self-similar solution faster than the continuos phase. This creates an 'overshoot' that reduces only at a position where the simulation sufficiently resolves the continuous phase velocity profile. From that point onwards the stochastic contribution decreases, and the solution becomes weakly dependent on $C_{o}$.

Using the dynamic model, the predicted levels of mixing and momentum layer thicknesses are significantly better than any single choice of static dispersion coefficients when the LES is well resolved. The ability of the dynamic model to predict correct levels of dispersion is impeded at very coarse grid spacings, where the testfilter width lies outside the inertial range. Even so, the dispersion statistics were similar to those produced by the static coefficient models.

Although it was used here in the model proposed by Bini and Jones, the dynamic model could, in theory, be extended to different choices of drift and diffusion coefficients. A possible application would be in Lagrangian Stochastic dispersion models of fluid particles, where the same underlying assumption about particle energy could be imposed. The main difference would be that in the latter case the dynamic coefficient would not be mass weighted since all fluid particles are assumed to carry the same weight. 


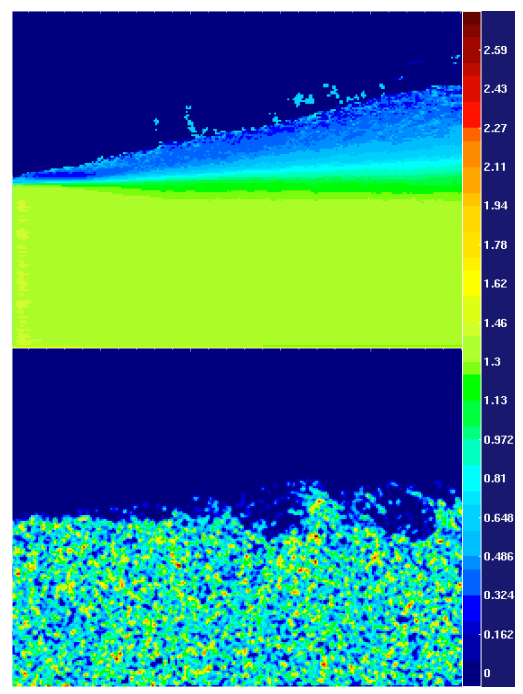

(a)

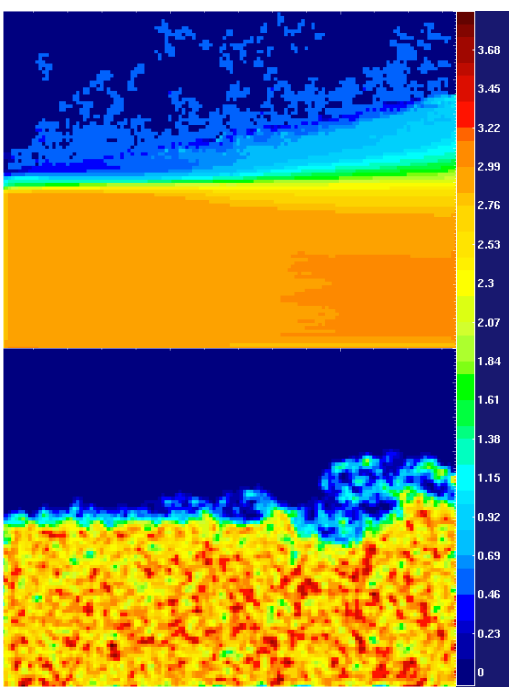

(b)

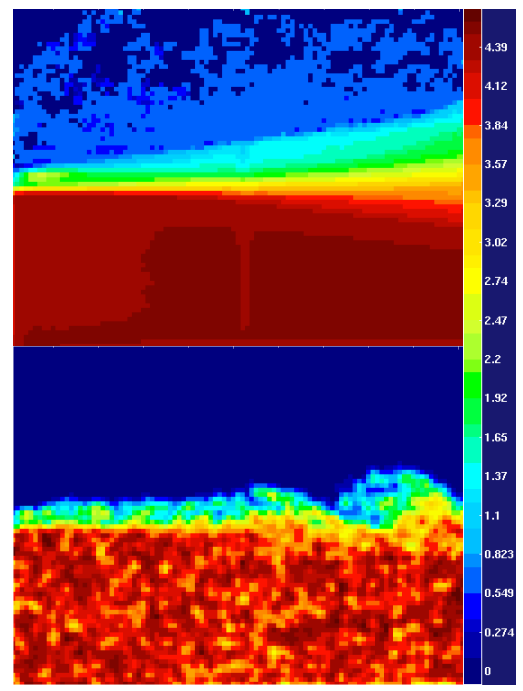

(c)

FIG. 15. Contour plots of ensemble-averaged (top) and instantaneous (bottom) dynamic dispersion coefficient for: (a) 0.8 mm, (b) $1.5 \mathrm{~mm}$, (c) $2 \mathrm{~mm}$ grid spacings.

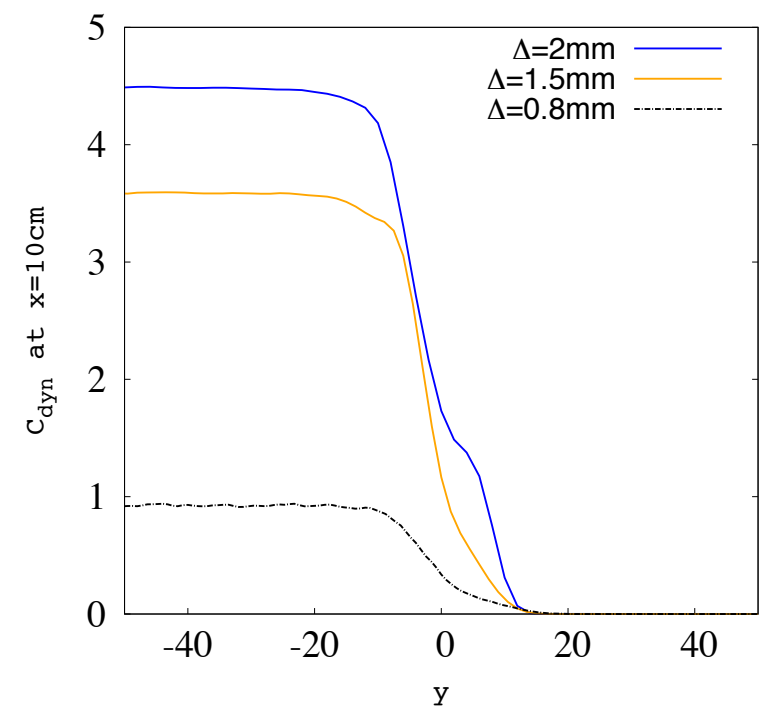

FIG. 16. Average dynamic coefficient versus height in the mixing layer for the three grid spacings investigated, at $x=$ $100 \mathrm{~mm} . C_{d y n}=0$ where particles do not exist.

Finally, the dynamic model offers advantages to the static equivalent. It does not require to choose the value of the static coefficient. It provides better results than the static coefficient when the only parameter varied is the particle size. Moreover, due to its coupling with the flow, it produces smoother results than static coefficients.

\section{ACKNOWLEDGMENT}

The financial support of the EPSRC through a DTA award is gratefully acknowledged.

${ }^{1}$ H. Kim and W. Chun, "The particle size effect on pollutant formation in pulverized coal combustion," Environ. Eng. Res., 3, 21-29 (1998).

${ }^{2}$ S. Mosbach, M. Celnik, A. Raj, M. Kraft, H. Zhang, and K. K. S. Kubo, "Towards a detailed soot model for internal combustion engines," Combustion and Flame, 156, 1156-1165 (2009).

${ }^{3}$ R. Wenglarz and R. Fox, "Physical aspects of deposition from coal-water fuels under gas turbine conditions," J. Eng. Gas Turbines Power, 112, 9-14 (1990).

${ }^{4}$ K. Ragland, M. Misra, D. Aerts, and C. Palmer, "Ash deposition in a wood-fired gas turbine," J. Eng. Gas Turbines Power, 117, 509-12 (1995).

${ }^{5}$ B. Lazaro and J. Lasheras, "Droplet dispersion and transport mechanisms in a turbulent, free shear-layer," Proc. Combust. Inst, 22, 1991-1998 (1989).

${ }^{6} \mathrm{~B}$. Lazaro and J. Lasheras, "Particle dispersion in a turbulent plane shear layer," Phys. Fluids, 1, 1035-1044 (1989).

${ }^{7} \mathrm{~B}$. Lazaro and J. Lasheras, "Particle dispersion in the developing free shear layer. part 1. unforced flow," J. Fluid Mech., 235, 143-178 (1992).

${ }^{8}$ B. Lazaro and J. Lasheras, "Particle dispersion in the developing free shear layer. part 2. forced flow," J. Fluid Mech., 235, 179221 (1992).

${ }^{9} \mathrm{~F}$. Yeh and U. Lei, "On the motion of small particles in a homogeneous isotropic turbulent flow," Phys. Fluids, 3, 2571-2586 (1991).

${ }^{10} \mathrm{~F}$. Toschi and E. Bodenschatz, "Lagrangian properties of particles in turbulence," Annual Review of Fluid Mechanics, 41, 375-404 (2009).

${ }^{11} \mathrm{~A}$. Guha, "Transport and deposition of particles in turbulent and laminar flow," Annu. Rev. Fluid Mech, 40, 311-341 (2008).

${ }^{12} \mathrm{~F}$. Menter and Y. Egorov, "The scale-adaptive simulation method for unsteady turbulent flow predictions. part 1: Theory and model description," Flow Turbulence Combust, 85, 112-138 (2010). 
${ }^{13} \mathrm{~J}$. Rotta, "über eine methode zur berechnung turbulenter scheströmungen," Aerodynamische Versuchsanstalt Rep., 69, A14 (1968).

${ }^{14} \mathrm{P}$. Moin and K. Mahesh, "Direct numerical simulation: A tool in turbulence research," Annu. Rev. Fluid Mech, 30, 539-78 (1998).

${ }^{15}$ A. Berrouk, D. Laurence, J. Riley, and D. Stock, "Stochastic modelling of inertial particle dispersion by subgrid motion for les of high reynolds number pipe flow," Journal of Turbulence, 8, N50 (2007).

${ }^{16} \mathrm{Q}$. Wang and K. D. Squires, "Large eddy simulation of particleladen turbulent channel flow," Phys. Fluids, 8, 1207 (1996).

${ }^{17}$ W. S. J. Uijttewaal and R. V. A. Oliemans, "Particle dispersion and deposition in direct numerical and large eddy simulations of vertical pipe flows," Phys. Fluids, 8, 2590 (1996).

${ }^{18}$ R. S. Miller and J. Bellan, "Direct numerical simulation and subgrid analysis of a transitional droplet laden mixing layer," Phys. Fluids, 12, 650 (2000)

${ }^{19}$ J. Kuerten and A. Vreman, "Can turbophoresis be predicted by large-eddy simulation?" Phys. Fluids, 17, 011701 (2005).

${ }^{20} \mathrm{P}$. Fede and O. Simonin, "Numerical study of the subgrid fluid turbulence effects on the statistics of heavy colliding particles," Phys. Fluids, 18, 045103 (2006).

${ }^{21}$ B. Shotorban and F. Mashayek, "A stochastic model for particle motion in large-eddy simulation," Journal of Turbulence, 7, N18 (2006).

${ }^{22}$ M. Bini and W. P. Jones, "Large eddy simulation of particle laden turbulent flows," J Fluid Mech, 614, 207-252 (2008).

${ }^{23}$ J. Pozorski and S. Apte, "Filtered particle tracking in isotropic turbulence and stochastic modeling of subgrid-scale dispersion," Int. J. Multiphase Flow, 35, 118-128 (2009).

${ }^{24}$ J. Pozorski, M. Knorps, and M. Luniewski, "Effects of subfilter velocity modelling on dispersed phase in les of heated channel flow," J. Phys.: Conf. Ser., 33, 012014 (2011).

${ }^{25}$ S. Stolz, N. A. Adams, and L. Kleiser, "An approximate deconvolution model for large-eddy simulation with application to incompressible wall- bounded flows," Phys. Fluids, 13, 997 (2001).

${ }^{26}$ J. Kuerten, "Subgrid modeling in particle-laden channel flow," Phys. FLuids, 18, 025108 (2006).

${ }^{27}$ B. Shotorban and F. Mashayek, "Modeling subgrid-scale effects on particles by approximate deconvolution," Phys. Fluids, 17, 081701 (2005).

${ }^{28}$ C. W. Gardiner, Handbook of Stochastic Methods for Physics, Chemistry and the Natural Sciences (Springer, 2003).

${ }^{29}$ J. D. Wilson and B. Sawford, "Review of lagrangian stochastic modles for trajectories in turbulent atmosphere," BoundaryLayer Meteorology, 78, 191-210 (1996).

${ }^{30} \mathrm{D}$. Haworth, "Progress in probability density function methods for turbulent reacting flows," Progress in Energy and Combustion Science, 36, 168-259 (2010).

${ }^{31}$ S. Pope, Turbulent Flows (Cambridge University Press, 2000).

${ }^{32}$ S. Pope, "Lagrangian pdf methods for turbulent flows," Annu. Rev. Fluid Mech, 26, 23-63 (1994).

${ }^{33} \mathrm{D}$. Thomson, "Criteria for the selection of stochastic models of particle trajectories in turbulent flows," J. Fluid Mech., 180, 529-556 (1987).

${ }^{34} \mathrm{~A}$. Kolmogorov, "The local structure of turbulence in incompressible viscous fluid for very larg reynolds numbers," Dolakady Akademii Nauk SSSR, 32, 299-303 (1941).

${ }^{35} \mathrm{R}$. Lien and E. D'Asaro, "The kolmogorov constant for the lagrangian velocity spectrum and structure function," Phys. Fluids, 14, 4456 (2002).

${ }^{36}$ G. Jin, G. He, L. Wang, and J. Zhang, "Subgrid scale fluid velocity timescales seen by inertial particles in large-eddy simulation of particle-laden turbulence," Int. J. Multiphase Flow, 36, 432-437 (2010)

${ }^{37}$ M. Bini, Large Eddy Simulation of particle and droplet laden flows with stochastic modelling of subfilter scales, Ph.D. thesis,
Imperial College London (2006)

${ }^{38} \mathrm{M}$. Bini and W. P. Jones, "Particle acceleration in turbulent flows: A class of nonlinear stochastic models for intermittency," Phys. Fluids, 19, 035104-1 (2007).

${ }^{39} \mathrm{~F}$. Williams, "Spray combustion and atomisation," Physics of Fluids, 1 n.6, 541-545 (1958)

${ }^{40}$ H. Risken, The Fokker Planck Equation (Springer, 1984).

${ }^{41}$ I. Klimontovich, The Statistical Theory of Non-equilibrium Processes in a Plasma (Pergamon, 1969).

${ }^{42} \mathrm{~S}$. Subramaniam, "Statistical representation of a spray as a point process," Phys. Fluids, 13, 2413-2431 (2000).

${ }^{43}$ W. Jones, S. Lyra, and S. Navarro-Martinez, "Large eddy simulation of a swirl stabilized spray flame," Proc. Combust. Inst., 33, 2153-2160 (2011).

${ }^{44} \mathrm{M}$. Maxey and J. Riley, "Equation of motion for a small rigid sphere in a nonuniform flow." Phys, 26, 883-889 (1983).

${ }^{45}$ N. Okong'o and J. Bellan, "Consistent large-eddy simulation of a temporal mixing layer with evaporating drops. part 1: Direct numerical simulation, formulation and a priori analysis," J. Fluid Mech., 499, 1-47 (2004).

${ }^{46}$ M. C. Yuen and L. W. Chen, "On drag of evaporating liquid droplets," Comb. Sci. \& Tech., 21, 537-542 (1976).

${ }^{47}$ D. K. Lilly, "A proposed modification of the germano subgridscale closure method," Physics of Fluids, 4, 633635 (1992).

${ }^{48} \mathrm{U}$. Piomelli and J. Liu, "Large-eddy simulation of rotating channel flow using localized dynamic model," Phys. Fluids, 7(4), 839848 (1995).

${ }^{49}$ R. Courant, K. Friedrichs, and H. Lewy, "On the partial difference equations of mathematical physics," Mathematische Annalen, 100, 32-74 (1928).

${ }^{50} \mathrm{~A}$. Ahmed and S. Elghobashi, "Direct numerical simulation of particle dispersion in homogeneous turbulent shear flows," Phys. Fluids, 13 (11), 3346 (2001)

${ }^{51}$ T. Gerz, U. Schumann, and S. Elghobashi, "Direct numerical simulation of stratified homogeneous turbulent shear flows," J. Fluid Mech., 200, 563 (1989).

${ }^{52} \mathrm{~A}$. Kempf, B. Geurts, and J. Ofelein, "Error analysis of largeeddy simulation of the turbulent non-premixed sydney bluff-body flame," Combustion and Flame, 158, 2408-2419 (2011).

${ }^{53}$ M. Pettit, B. Coriton, A. Gomez, and A. Kempf, "Large eddy simulation and experiments on non-premixed highly turbulent opposed jet flows," Proc. Combust. Inst, 33, 1391-1399 (2011).

${ }^{54} \mathrm{~S}$. Chumakov, "Scaling properties of subgrid-scale energy dissipation," Phys. Fluids, 19, 058104 (2007).

${ }^{55} \mathrm{~J}$. Smagorinsky, "General circulation experiments with the primitive equations, i. the basic experiment." Monthly Weather Review, 152, 9199 (1963).

${ }^{56}$ D. Lilly, "The representation of small-scale turbulence in numerical simulation experiments," Proc. of the IBM Scientific Computing Symp. on Environmental Sciences, 1, 195-210 (1967).

${ }^{57}$ W. Jones, S. Lyra, and A. Marquis, "Large eddy simulation of a droplet laden turbulent mixing layer," International Journal of Heat and Fluid Flow, 31, 93-100 (2010).

${ }^{58} \mathrm{M}$. T. B. Cetegena, "Development of mixing and dispersion in an isothermal, droplet-laden, confined turbulent mixing layer," Combustion Science and Technology, 130, 131-169 (1997).

${ }^{59}$ A. Kempf, M. Klein, and J. Janicka, "Efficient generation of initial and inflow conditions for transient turbulent flows in arbitrary geometries," Flow, Turbulence and Combustion, 74, 67-84 (2005).

${ }^{60}$ M. Klein, A. Sadiki, and J. Janicka, "A digital filter based generation of inflow data for spatially developing direct numerical or large eddy simulations," Journal of Computational Physics, 186, 652-665 (2003). 